Western University

Scholarship@Western

$3-5-2020$

\title{
Expression Changes Confirm Genomic Variants Predicted to Result in Allele-Specific, Alternative mRNA Splicing
}

Peter Rogan

The University of Western Ontario, progan@uwo.ca

Follow this and additional works at: https://ir.lib.uwo.ca/biochempub

Part of the Biochemistry Commons

Citation of this paper:

Rogan, Peter, "Expression Changes Confirm Genomic Variants Predicted to Result in Allele-Specific, Alternative mRNA Splicing" (2020). Biochemistry Publications. 195.

https://ir.lib.uwo.ca/biochempub/195 


\section{OPEN ACCESS}

Edited by:

Emanuele Buratti International Centre for Genetic Engineering and Biotechnology,

Italy

Reviewed by: Stefan Stamm,

University of Kentucky,

United States

Lucie Grodecká, Center of Cardiovascular and Transplant Surgery, Czechia

*Correspondence: Peter K. Rogan progan@uwo.ca

Specialty section: This article was submitted to RNA, a section of the journal

Frontiers in Genetics

Received: 13 September 2019 Accepted: 30 January 2020 Published: 05 March 2020

Citation:

Mucaki EJ, Shirley BC and Rogan PK (2020) Expression Changes Confirm Genomic

Variants Predicted to

Result in Allele-Specific, Alternative mRNA Splicing.

Front. Genet. 11:109. doi: 10.3389/fgene.2020.00109
Expression Changes Confirm Genomic Variants Predicted to Result in Allele-Specific, Alternative mRNA Splicing

\author{
Eliseos J. Mucaki ${ }^{1}$, Ben C. Shirley ${ }^{2}$ and Peter K. Rogan ${ }^{1,2,3,4 *}$ \\ 1 Department of Biochemistry, University of Western Ontario, London, ON, Canada, ${ }^{2}$ CytoGnomix, London, ON, Canada, \\ ${ }^{3}$ Department of Oncology University of Western Ontario, London, ON, Canada, ${ }^{4}$ Department of Computer Science, \\ University of Western Ontario, London, ON, Canada
}

Splice isoform structure and abundance can be affected by either noncoding or masquerading coding variants that alter the structure or abundance of transcripts. When these variants are common in the population, these nonconstitutive transcripts are sufficiently frequent so as to resemble naturally occurring, alternative mRNA splicing. Prediction of the effects of such variants has been shown to be accurate using information theory-based methods. Single nucleotide polymorphisms (SNPs) predicted to significantly alter natural and/or cryptic splice site strength were shown to affect gene expression. Splicing changes for known SNP genotypes were confirmed in HapMap lymphoblastoid cell lines with gene expression microarrays and custom designed q-RT-PCR or TaqMan assays. The majority of these SNPs (15 of 22) as well as an independent set of 24 variants were then subjected to RNAseq analysis using the ValidSpliceMut web beacon (http:// validsplicemut.cytognomix.com), which is based on data from the Cancer Genome Atlas and International Cancer Genome Consortium. SNPs from different genes analyzed with gene expression microarray and q-RT-PCR exhibited significant changes in affected splice site use. Thirteen SNPs directly affected exon inclusion and 10 altered cryptic site use. Homozygous SNP genotypes resulting in stronger splice sites exhibited higher levels of processed mRNA than alleles associated with weaker sites. Four SNPs exhibited variable expression among individuals with the same genotypes, masking statistically significant expression differences between alleles. Genome-wide information theory and expression analyses (RNAseq) in tumor exomes and genomes confirmed splicing effects for 7 of the HapMap SNP and 14 SNPs identified from tumor genomes. q-RT-PCR resolved rare splice isoforms with read abundance too low for statistical significance in ValidSpliceMut. Nevertheless, the web-beacon provides evidence of unanticipated splicing outcomes, for example, intron retention due to compromised recognition of constitutive splice sites. Thus, ValidSpliceMut and q-RT-PCR represent complementary resources for identification of allele-specific, alternative splicing.

Keywords: allele-specific gene expression, mRNA splicing, single nucleotide polymorphism, mutation, cryptic splicing, intron retention, alternative splicing, information theory 


\section{INTRODUCTION}

Accurate and comprehensive methods are needed for predicting impact of noncoding mutations, in particular, mRNA splicing defects, which are prevalent in genetic disease (Krawczak et al., 1992; Teraoka et al., 1999; Ars et al., 2003; Spielmann and Mundlos, 2016; Gloss and Dinger, 2018). This class of mutations may account for as much as $62 \%$ of point mutations (López-Bigas et al., 2005). Large transcriptome studies have suggested that a large fraction of genome-wide association studies (GWAS) signals for disease and complex traits are due to single nucleotide polymorphisms (SNPs) affecting mRNA splicing (Park et al., 2018). ValidSpliceMut (Shirley et al., 2019) presents evidence of altered splicing (Viner et al., 2014; Dorman et al., 2014) for 309,848 validated genome splice-variant predictions (Shirley et al., 2013). The majority of mutations were associated with exon skipping, cryptic site use, or intron retention, and in these cases ValidSpliceMut assigns a molecular phenotype classification to all variants as either aberrant, likely aberrant or inducing alternative isoforms.

While allele-specific alternative splicing can predispose for disease susceptibility (Park et al., 2018), these genetic variations also are associated with common phenotypic variability in populations (Hull et al., 2007). Soemedi et al. (2017) determined that $10 \%$ of a set of published disease-causing exonic mutations $(\mathrm{N}=4,964)$ altered splicing. Their analysis of a control set of exonic SNPs common among those without disease phenotypes revealed a smaller proportion $(3 \%)$ that altered splicing $(\mathrm{N}=228)$. However, we recently showed that splice-altering, common SNPs are considerably more abundant in tumor genomes in the ValidSpliceMut web-beacon (http://validsplicemut.cytognomix. com; Shirley et al., 2019). Variants with higher germline population frequencies which impact splicing are less likely than rare mutations with direct splicing effects to be involved in Mendelian diseases or cancer. The present study analyzes predicted splice-altering polymorphic variants in genotyped lymphoblastoid cell lines by q-RT-PCR, expression microarrays of samples of known SNP genotypes, and high throughput expression data corresponding to sequenced tumor exomes and genomes. The relatively high frequencies of these variants enable comparisons of expressed transcripts in multiple individuals and genotypes. Effects of these SNPs are confirmed by multiple methods, although the supporting evidence from these distinct approaches is often complementary, rather than entirely concordant.

An estimated $90 \%$ to $95 \%$ of all multiexon genes are alternatively spliced (Pan et al., 2008; Wang et al., 2008; Baralle and Giudice, 2017). The selection of splicing signals involves exon and intron sequences, complementarity with snRNAs, RNA secondary structure, and competition between spliceosomal recognition sites (Moore and Sharp, 1993; Berget, 1995; Park et al., 2018). U1 snRNP interacts with the donor (or 5') splice site (Zhuang and Weiner, 1986; Séraphin et al., 1988) and U2 (and U6) snRNP with the acceptor and branch sites of pre-mRNA (Parker et al., 1987; Wu and Manley, 1989). The majority of human splice donors (5') and acceptors (3') base pair with the U1 and U2 RNAs in spliceosomes, but are generally not precisely complementary to these sequences (Rogan et al., 2003). Additional exonic and intronic cis-regulatory elements can promote or suppress splice site recognition through recruitment of trans-acting splicing factors. SR proteins are positive trans-acting splicing factors which contain RNArecognition motifs (RRM) and a carboxy-terminal domain enriched in Arg/Ser dipeptides (SR domain; Birney et al., 1993). Binding of RRMs in pre-mRNA enhances exon recognition by promoting interactions with spliceosomal and other proteins (Fu and Maniatis, 1992). SR proteins function in splice site communication by forming an intron bridge needed for exon recognition (Zuo and Maniatis, 1996). Factors that negatively impact splicing include heterogeneous nuclear ribonucleoproteins (hnRNPs; Martinez-Contreras et al., 2007).

Splicing mutations affect normal exon recognition by altering the strengths of natural donor or acceptor sites and proximate cryptic sites, either independently or simultaneously. Weakened splice sites reduce of kinetics of mRNA processing, leading to an overall decrease in full length transcripts, increased exon skipping, cryptic splice site activation within exons or within adjacent introns, intron retention, and inclusion of cryptic, pseudo-exons (Talerico and Berget, 1990; Carothers et al., 1993; Buratti et al., 2006; Park et al., 2018). The kinetics of splicing at weaker cryptic sites is also slower than at natural sites (Domenjoud et al., 1993). Mutations strengthen cryptic sites either by increasing resemblance to "consensus sequences" (Nelson and Green, 1990) or by modulating the levels of SR proteins contributing to splice site recognition (Mayeda and Krainer, 1992; Cáceres et al., 1994). Mutations affecting splicing regulatory elements (Dietz et al., 1993; Richard and Beckmann, 1995) disrupt trans-acting SR protein interactions (Staknis and Reed, 1994) with distinct exonic and intronic cis-regulatory elements (Black, 2003).

Information theory-based (IT-based) models of donor and acceptor mRNA splice sites reveal the effects of changes in strengths of individual sites (termed $R_{i}$; Rogan et al., 1998; Rogan et al., 2003). This facilitates prediction of phenotypic severity (Rogan and Schneider, 1995; von Kodolitsch et al., 1999; von Kodolitsch et al., 2006). The effects of splicing mutations can be predicted in silico by information theory (Rogan and Schneider, 1995; Kannabiran et al., 1998; Rogan et al., 1998; Svojanovsky et al., 2000; Rogan et al., 2003; Caminsky et al., 2014; Dorman et al., 2014; Viner et al., 2014; Caminsky et al., 2016; Mucaki et al., 2016; Shirley et al., 2019), and these predictions can be confirmed by in vitro experimental studies (Vockley et al., 2000; Lamba et al., 2003; Rogan et al., 2003; Khan et al., 2004; Susani et al., 2004; Hobson et al., 2006; Caux-Moncoutier et al., 2009; Olsen et al., 2014; Vemula et al., 2014; Peterlongo et al., 2015). Strengths of one or more splice sites may be altered and, in some instances, concomitant with amino acid changes in coding sequences (Rogan et al., 1998; Peterlongo et al., 2015). Information analysis has been a successful approach for recognizing nondeleterious, sometimes polymorphic variants (Rogan and Schneider, 1995; Colombo et al., 2013), and for distinguishing of milder from severe mutations (Rogan et al., 1998; von Kodolitsch et al., 1999; Lacroix et al., 2012). 
Predicting the relative abundance of various transcripts by information analysis requires integration of the contributions of all pertinent cis-acting regulatory elements. We have applied quantitative methods to prioritize inferences as to which SNPs impact gene expression levels and transcript structure. Effects of mutations on combinations of splicing signals reveal changes in isoform structure and abundance (Mucaki et al., 2013; Caminsky et al., 2014). Multisite information theory-based models have also been used to detect and analyze SNP effects on cis-acting promoter modules that contribute to establishing transcript levels (Bi and Rogan, 2004; Vyhlidal et al., 2004; Lu et al., 2017; Lu and Rogan, 2019).

The robustness of this approach for predicting rare, deleterious splicing mutations justifies efforts to identify common SNPs that impact mRNA splicing. We previously described SNPs from dbSNP that affect splicing (Rogan et al., 1998; Nalla and Rogan, 2005). Here, we explicitly predict and validate SNPs that influence mRNA structure and levels of expression of the genes containing them in immortalized lymphoblastoid cell lines and tumors. Since constitutive splicing mutations can arise at other locations within premRNA sequences that elicit cryptic splicing, we examined whether more common genomic polymorphisms might frequently affect the abundance and structure of splice isoforms.

\section{METHODS}

\section{Information Analysis}

The protein-nucleic acid interactions intrinsic to splicing can be analyzed using information theory, which comprehensively and quantitatively models functional sequence variation based on a thermodynamic framework (Schneider, 1997). Donor and acceptor splice site strength can be predicted by the use of ITbased weight matrices derived from known functional sites (Rogan et al., 2003). The Automated Splice Site and Exon Definition server (ASSEDA) is an online resource based on the hg19 coordinate system to determine splice site information changes associated with genetic diseases (Mucaki et al., 2013). ASSEDA is now part of the MutationForecaster (http://www. mutationforecaster.com) variant interpretation system.

\section{Creation of Exon Array Database}

Exon-level microarrays have been used to compare abnormal expression for different cellular states, which can then be confirmed by q-RT-PCR (Thorsen et al., 2008). We hypothesized that the predicted effect of SNPs on expression of the proximate exon would correspond to the expression of exon microarray probes of genotyped individuals in the HapMap cohort. We used the dose-dependent expression of the minor allele to qualify SNPs for subsequent information analysis consistent with alterations of mRNA splicing. Additional SNPs predicted by information analysis were also tested for effects on splicing (Nalla and Rogan, 2005).

Expression data were normalized using the PLIER (Probe Logarithmic Intensity Error) method on Affymetrix Human
Exon 1.0 ST microarray data for 176 genotyped HapMap cell lines (Huang et al., 2007, Gene Expression Omnibus accession no. GSE 7792; Nembaware et al., 2008). Microarray probes which overlap SNPs, that were subsequently removed, were identified by intersecting dbSNP129 with probe coordinates [obtained from X:MAP (Yates et al., 2008) using the Galaxy Browser (Giardine et al., 2005)]. A MySQL database containing the PLIER normalized intensities and CEU (Utah residents with Northern and Western European ancestry) and YRI (Yoruba in Ibadan) genotypes for Phase I+II HapMap SNPs was created. Tables were derived to link SNPs to their nearest like-stranded probeset (to within $500 \mathrm{nt}$ ), and to associate probesets to the exons they may overlap (transcript and exon tables from Ensembl version 51). A MySQL query was used to create a table containing the splicing index (SI; intensity of a probeset divided by the overall gene intensity) of each probeset for each HapMap individual.

The database was queried to identify significant SI changes of an exonic probeset based on the genotype of a SNP the probeset was associated with (SNP within natural donor/acceptor region of exon). Probesets displaying a stepwise change in mean SI (where the mean SI of the heterozygous group is in between the mean SI values of the two homozygous groups) were identified using a different program script (criteria: the mean SI of homozygous rare and heterozygous groups are $<90 \%$ of the homozygous common group). Splicing Index boxplots were created with $\mathrm{R}$, where the $\mathrm{x}$ - and $\mathrm{y}$-axis are genotype and SI, respectively (Supplementary Image 1). These boxplots analyze the effect a SNP has on a particular probeset across all individuals.

SNPs with effects on splicing were validated by q-RT-PCR of lymphoblastoid cell lines. Where available, results were also compared to abnormal splicing patterns present in RNAseq data from tumors carrying these same SNPs (in the ValidSpliceMut database; Shirley et al., 2019). SNPs predicted to exhibit nominal effects on splicing $\left(\Delta R_{i}<1\right.$ bit $)$ were included to determine minimal detectable changes by q-RT-PCR.

\section{Cell Culture and RNA Extraction}

EBV-transformed lymphoblastoid cell lines of HapMap individuals with our SNPs of interest (homozygous common, heterozygous and homozygous rare when available) were ordered from the Coriell Cell Repositories (CEU: GM07000, GM07019, GM07022, GM07056, GM11992, GM11994, GM11995, GM12872; YRI: GM18855, GM18858, GM18859, GM18860, GM19092, GM19093, GM19094, GM19140, GM19159). Cells were grown in HyClone RPMI-1640 medium [15\% FBS (HyClone), 1\% L-Glutamine, and 1\% Penicillin: streptomycin (Invitrogen); $37^{\circ} \mathrm{C}, 5 \% \mathrm{CO}_{2}$ ]. RNA was extracted with Trizol LS (Invitrogen) from $10^{6}$ cells and treated with DNAase [20 mM $\mathrm{MgCl}_{2}$ (Invitrogen), $2 \mathrm{mM}$ DTT (SigmaAldrich), $0.4 \mathrm{U} / \mu \mathrm{L}$ RNasin (Promega), $10 \mu \mathrm{g} / \mathrm{ml}$ DNase (Worthington Biochemical) in $1 \mathrm{x}$ TE buffer] at $37^{\circ} \mathrm{C}$ for 15 min. The reaction was stopped with EDTA $(0.05 \mathrm{M} ; 2.5 \% \mathrm{v} / \mathrm{v})$, and heated to $65^{\circ} \mathrm{C}$ for $20 \mathrm{~min}$, followed by ethanol precipitation (resuspended in $0.1 \% \mathrm{v} / \mathrm{v}$ DEPC-treated $1 \mathrm{x}$ TE buffer). DNA was extracted using a Puregene Tissue Core Kit B (Qiagen). 


\section{Design of Real-Time Expression Assays}

Sequences were obtained from UCSC and Ensembl. DNA primers used to amplify a known splice form, or one predicted by information analysis, were designed using Primer Express (ABI). DNA primers (Supplementary Table 1) were obtained from IDT (Coralville, IA, USA), and dissolved to $200 \mathrm{uM}$. Primers were placed over junctions of interest to amplify a single splice form. $\mathrm{T}_{\mathrm{m}}$ ranged from $58^{\circ} \mathrm{C}-65^{\circ} \mathrm{C}$, and amplicon lengths varied from 69-136 nt. BLASTn (Refseq_RNA database) was used to reduce possible cross-hybridization. Primers were designed to amplify the wildtype splice form, exon skipping (if a natural site is weakened), and cryptic site splice forms which were either previously reported (UCSC mRNA and EST tracks) or predicted by information analysis (where $R_{i}$ cryptic site $\geq R_{i}$ weakened natural site).

Two types of reference amplicons were used to quantify allele specific splice forms. These consisted of intrinsic products derived from constitutively spliced exons with the same gene and external genes with high uniformity of expression among HapMap cell lines. Reference primers internal to the genes of interest were designed 1-4 exons adjacent from the affected exon (exons without any evidence of variation in the UCSC Genome Browser; Kent et al., 2002), placed upstream of the SNP of interest whenever possible. Two advantages to including an internal reference in the q-RT-PCR experiment include: potential detection of changes in total mRNA levels; and account for inter-individual variation of expression.

External reference genes (excluding the SNP of interest) were chosen based on consistent PLIER intensities with low coefficients of variation in expression among all 176 HapMap individuals. The following external controls were selected: exon 39 of SI (PLIER intensity $11.4 \pm 1.7)$, exon 9 of FRMPD1 (22 \pm 2.81), exon 46 of DNAH1 (78.5 \pm 9.54$)$, exon 3 of CCDC137 $(224 \pm 25)$, and exon 25 of VPS39 (497 \pm 76$)$. The external reference chosen for an experiment was matched to the intensity of the probeset within the exon of interest. This decreased potential errors in $\Delta \Delta \mathrm{C}_{\mathrm{T}}$ values and proved to be accurate and reproducible for most genes.

To control for interindividual variation in expression, we compared expression in HapMap individuals based on their SNP genotypes and familial relatedness. Families with all three possible genotypes were available (homozygous common, rare, and heterozygous) for 12 of these SNPs (rs1805377, rs2243187, rs2070573, rs2835655, rs2835585, rs2072049, rs1893592, rs6003906, rs1018448, rs13076750, rs16802, and rs8130564). For those families in which all genotypes were not represented, samples from the same ethnic background (YRI or CEU populations) were compared for the missing genotype $(\mathrm{N}=8$; rs17002806, rs2266988, rs1333973, rs743920, rs2285141, rs2838010, rs10190751, rs16994182; individuals with homozygous common and rare genotypes were from the same families for the latter two SNPs). Two SNPs were tested using homozygous individuals from different ethnic backgrounds: rs3747107 (GUSBP11) and rs2252576 (BACE2). While the splicing impact of rs3747107 was clearly observable by q-RT-PCR, either background or data noise did impact the interpretation of effects of rs2252576.

\section{PCR and Quantitative RT-PCR}

M-MLV reverse transcriptase (Invitrogen) converted $1 \mu \mathrm{g}$ of DNase-treated RNA to cDNA with 20 nt Oligo-dT $(25 \mu \mathrm{g} / \mathrm{ml}$; IDT) and rRNAsin (Promega). Precipitated cDNA was resuspended in water at $20 \mathrm{ng} / \mu \mathrm{l}$ of original RNA concentration. All designed primer sets were tested with conventional PCR to ensure a single product at the expected size. PCR reactions were prepared with 1.0 M Betaine (SigmaAldrich), and were heated to $80^{\circ} \mathrm{C}$ before adding Taq Polymerase (Invitrogen). Optimal $\mathrm{T}_{\mathrm{m}}$ for each primer set was determined to obtain maximum yield.

Quantitative PCR was performed with an Eppendorf Mastercycler ep Realplex 4, a Bio-Rad CFX96, as well as a Stratagene Mx3005P. SYBR Green assays were performed using the KAPA SYBR FAST qPCR kit (Kapa Biosystems) in $10 \mu \mathrm{l}$ reactions using $200 \mu \mathrm{M}$ of each primer and $24 \mathrm{ng}$ total of cDNA per reaction. For some tests, SsoFast EvaGreen supermix (Bio-Rad) was used with $500 \mu \mathrm{M}$ of each primer instead.

When testing the effect of a SNP, amplification reactions with all primers designed to detect all relevant isoforms (as well as the gene internal reference and external reference) were run simultaneously, in triplicate. $C_{t}$ values obtained from these experiments were normalized against the same external reference using the Relative Expression Software Tool (REST; http://www.gene-quantification.de/rest.html; Pfaffl et al., 2002).

\section{Taqman Assay}

Two dual-labeled Taqman probes were designed to detect the two splice forms of XRCC4 (detecting alternative forms of exon 8 either with or without a $6 \mathrm{nt}$ deletion at the $5^{\prime}$ end). Probes were placed over the sequence junction of interest where variation would be near the probe middle (Supplementary Table 1). The assay was performed on an ABI StepOne Real-Time PCR system using ABI Genotyping Master Mix. Experiment was run in $25 \mu \mathrm{l}$ reactions (300 nM each primer, $400 \mathrm{nM}$ probe [5'-FAM or TET fluorophore with a 3' Black Hole quencher; IDT], and $80 \mathrm{ng}$ cDNA total). Probes were tested in separate reactions.

\section{RNAseq Analyses}

The previous analyses were extended to include 24 additional, common SNPs for their potential influence on splicing. All SNVs present in ICGC (International Cancer Genome Consortium) patients (Shirley et al., 2019) were evaluated by the Shannon Pipeline (SP; Shirley et al., 2013) to identify those altering splice site strength. Common SNPs (average heterozygosity $>10 \%$ in dbSNP 150) predicted to decrease natural splice site strength by SP (where $\Delta R_{i}<-1$ bit) were selected. ICGC patients carrying these flagged SNPs were identified, and the expression of the corresponding SNP-containing region in RNAseq was visualized with IGV (Integrated Genome Viewer; https://igv.org; Robinson et al., 2017). Similar RNAseq reads were grouped using IGV collapse and sort commands, which caused nonconstitutive spliced reads to cosegregate to the top of the viewing window. IGV images which did not meet our gene expression criteria (exon affected by the SNP must have $\geq 5$ RNAseq reads present) were eliminated. As this generated thousands of images, we 
report the analysis of two ICGC patients [DO47132 (Renal Cell Cancer) and DO52711 (Chronic Lymphocytic Leukemia)], chosen randomly, preselecting tissues to increase the likelihood of finding expression in these regions. Images were evaluated sequentially (in order of rsID value) and only concluded once the first 24 SNPs meeting these criteria were found. This type of analysis could not reveal a splicing event to be more abundant in these patients when compared to noncarriers. Nevertheless, splicing information changes resulting from SNPs corresponded to observed alternative and/or other novel splice isoforms. We then queried the ValidSpliceMut database for these SNPs, as abnormal splicing was only flagged in the database when the junction-read or read-abundance counts significantly exceeded corresponding evidence type in a large set of normal control samples (Shirley et al., 2019).

\section{RESULTS}

\section{Selection of Candidate SNPs Affecting Splicing}

A publicly available exon microarray dataset was initially used to locate exons affected by SNPs altering splice site strength. A change in the mean SI of a diagnostic probeset in individuals of differing genotypes at the same variant can suggest altered splicing. The increase or decrease in SI is related to the expected impact of the SNP on splicing. For example, an exonic probe which detects a normally spliced mRNA will have decreased SI in the event of skipping. Mean SI may be increased when a probe detects the use of an intronic cryptic splice site. SNPs with strong impact on splicing will distinguish mean SI levels of individuals homozygous for the major versus minor alleles (and with heterozygous genotypes).

There were 9,328 HapMap-annotated SNPs within donor/ acceptor regions of known exons which contained at least one probeset. Of 987 SNPs that are associated to exonic probesets which differ in mean SI between the homozygous common and rare HapMap individuals, 573 caused a decrease in natural site $R_{i}$ value. Inactivating and leaky splicing variants (reduction in information content where final $R_{i} \geq R_{i, \text { minimum }}$ [minimum functional splice site strength]) both exhibit reduced SI values and were similarly abundant. Thus, both severe and moderate splicing mutations with reduced penetrance and milder molecular phenotypes were detected, consistent with Mendelian disorders (von Kodolitsch et al., 1999; von Kodolitsch et al., 2006).

Of the SNPs associated with significant changes in $R_{i}$ (termed $\left.\Delta R_{i}\right), 9,328$ occurred within the natural splice sites of exons detectable with microarray probesets. We initially focused on 21 SNPs on chromosome 21 ( $0.23 \%$ total, $18.8 \%$ of chr 21$)$ and 34 on chromosome $22(0.36 \%$ of total, $14.5 \%$ of chr22) associated with stepwise decreases in probeset intensity at each genotype. Seven of the chr21 SNPs and nine of the chr22 SNPs caused information changes with either natural splice site $\Delta R_{i} \geq 0.1$ bits, or cryptic site(s) with an $R_{i}$ value comparable to a neighbouring natural site, and in which mRNA or EST data supported use of the cryptic site. These SNPs included: rs2075276 [MGC16703], rs2838010 [FAM3B], rs3747107 [GUSBP11], rs2070573 [C21orf2], rs17002806 [WBP2NL], rs3950176 [EMID1], rs1018448 [ARFGAP3], rs6003906 [DERL3], rs2266988 [PRAME], rs2072049 [PRAME], rs2285141 [CYB5R3], rs2252576 [BACE2], rs16802 [BCR], rs 17357592 [COL6A2], rs 16994182 [CLDN14], and rs8130564 [TMPRSS3].

The minimum information change for detecting a splicing effect by expression microarray is constrained by several factors. Detection of splice isoforms can be limited by genomic probeset coverage, which cannot distinguish alternative splicing events in close proximity (see Figure 1A). Even where genotype-directed SI changes are very distinct, some individuals with the common allele have equivalent SI values to individuals with the rare allele [rs2070573 (Figure 2) and rs1333973 (Figure 3)]. In some cases, the number of individuals with a particular genotype is insufficient for statistical significance (rs2243187; Supplementary Image 1.4). Although exon microarrays can be used to find potential alternate splicing and give support to our predictions, it became necessary to validate the microarray predictions by q-RT-PCR, TaqMan assays, and with RNAseq data from SNP carriers.

We report q-RT-PCR validation studies for 13 of the 16 SNPs (q-RT-PCR primers could not be designed for rs16994182, rs2075276, and rs3950176), along with nine other candidate SNPs from our previous information theory-based analyses (Nalla and Rogan, 2005): rs1805377 [XRCC4], rs2243187 [IL19], rs2835585 [TTC3], rs2865655 [TTC3], rs1893592 [UBASH3A], rs743920 [EMID1], rs13076750 [LPP], rs1333973 [IFI44L], and rs10190751 [CFLAR].

After amplification of known and predicted splice forms (Supplementary Table 1), 15 SNPs showed measurable changes in splicing consistent with information-theory predictions. Ten increase alternate splice site use (two of which increased strength of cryptic site, eight activated an unaffected pre-existing cryptic site), six affect exon inclusion (five increased exon skipping), three increased activation of an alternative exon, and four decreased overall expression levels. Altered splicing could not be validated for six SNPs, however experimental analyses of three of the five SNPs where $\Delta R_{i}<1$ bit were hampered by high interindividual variability in expression.

Changes in splice site information were used to predict observed differences in splice isoform levels (Table 1). Figures 1-3 and Supplementary Image $\mathbf{1}$ indicate the experimentally-determined splicing effects for each SNP, a modified UCSC Genome Browser image of the relevant region, boxplots showing exon microarray expression levels of each allele for the relevant probesets, and an IGV image of the RNAseq results for an individual tumor carrying the SNP. Abundance of the aberrant splice forms measured by q-RTPCR (relative to an internal gene reference) is indicated in Table 2. Changes in predicted splice site strength were consistent with results measured by q-RT-PCR for 12 out of the 15 SNP (exceptions were rs2070573, rs17002806, and rs2835585). Variants predicted to reduce strength $\geq 100$-fold 
A

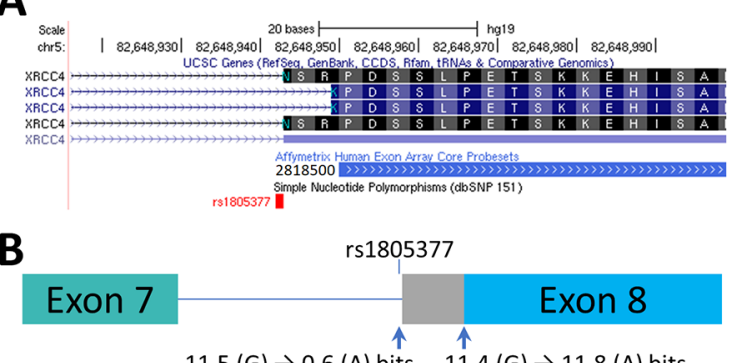

$11.5(\mathrm{G}) \rightarrow 0.6(\mathrm{~A})$ bits $\quad 11.4(\mathrm{G}) \rightarrow 11.8(\mathrm{~A})$ bits
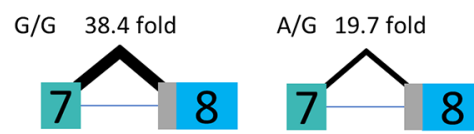

A/A 1 fold

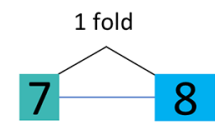

C

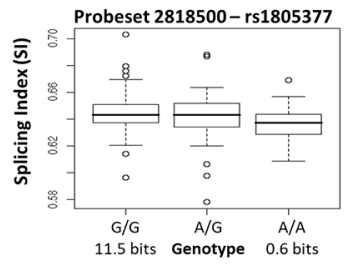

D

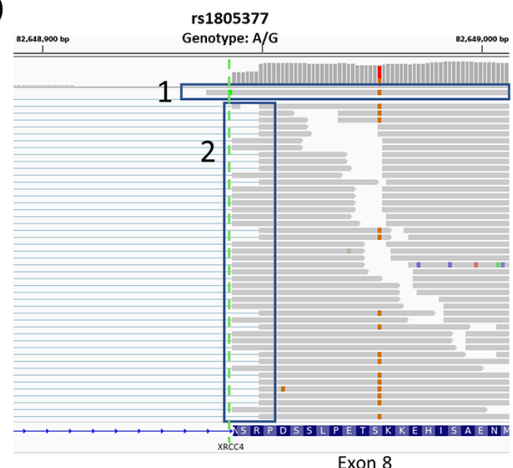

FIGURE 1 | Splicing Impact of rs1805377 (XRCC4). The natural acceptor of XRCC4 exon 8 is abolished by rs1805377 (11.5 -> 0.6 bits) while simultaneously strengthening a second exonic cryptic acceptor 6nt downstream (11.4 to 11.8 bits), resulting in a 6nt deletion in the mRNA. (A) Both of these acceptor sites have been validated in GenBank mRNAs, i.e., NM_022406 and NM_003401 (UCSC panel derived from http://genome.ucsc.edu). (B) The relative abundance of the two splice forms was determined by q-RT-PCR. The weaker rs 1805377 A/A genotype ( 0.6 bit acceptor) was used $\sim 47$-fold less frequently than the cryptic downstream acceptor (11.8 bits). (C) The two splice isoforms cannot be distinguished by the exon microarray as the upstream probeset (ID 2818500) does not overlap the variable region, though the average expression of the rs1805377 A/A genotype is reduced. (D) ValidSpliceMut flagged this mutation for intron retention, which can be observed in the RNAseq of heterozygous ICGC patient DO27779 [Box 1]. Use of both acceptor sites is also evident [Box 2]. For more detail for this and all of the other single nucleotide polymorphisms (SNPs) analyzed, refer to Supplementary Image 1.
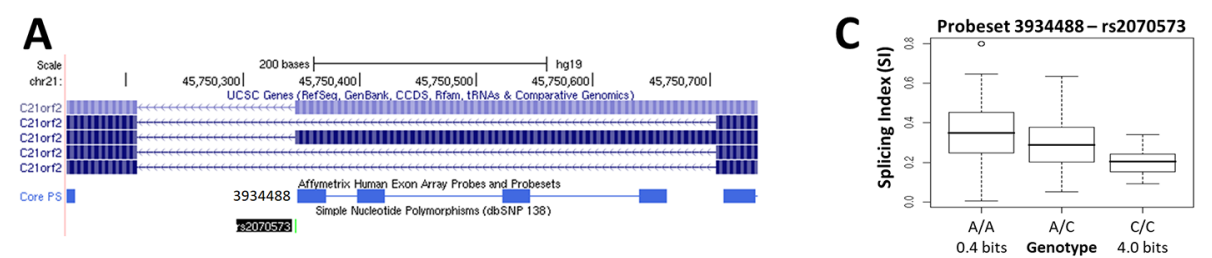

B

D
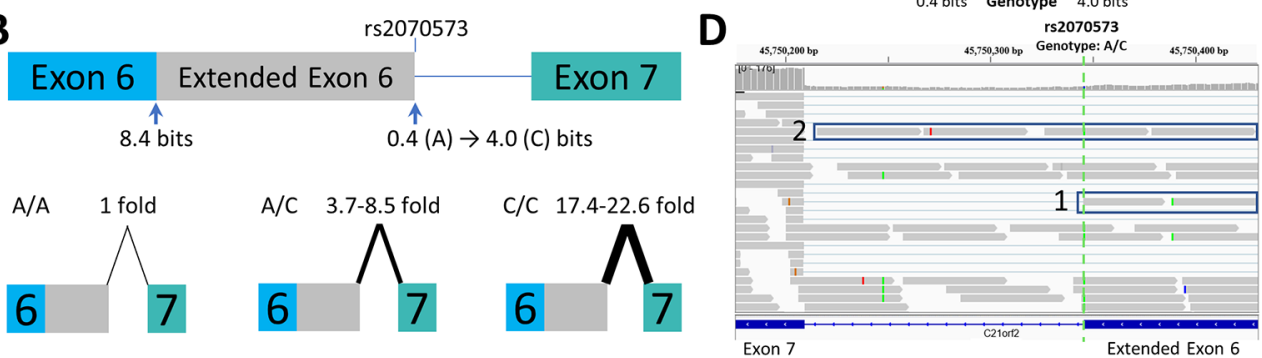

FIGURE 2 | Splicing Impact of rs2070573 (C210rf2). (A) The single nucleotide polymorphisms (SNP) rs2070573 is a common polymorphism which alters the first nucleotide of the extended form of C21orf2 exon 6. (B) The donor site is strengthened by the presence of the C-allele $\left(R_{i} 0.4\right.$ to 4.0 bits; $\left.\mathrm{A}>\mathrm{C}\right)$ and its use extends the exon by $360 \mathrm{nt}$. Q-RT-PCR found a 4-9-fold and 17-23-fold increase in the extended exon 6 splice form in the A/C and C/C cell lines tested, respectively. (C) The exon microarray probeset which detects the extension (ID 3934488) shows a stepwise increase in SI with C-allele individuals which supports the q-RT-PCR result. (D) The variant was present in ValidSpliceMut, which associated the A-allele with an increase in total intron retention [six patients flagged for total intron retention read abundance; $\mathrm{p}=0.019$ (average over all patients)]. This image displays sequence read distributions in the RNAseq data of TCGA BRCA patient, TCGA$\mathrm{BH}-\mathrm{AOHO}$, who is heterozygous for rs2070573. The IGV panel indicates reads corresponding to total intron 6 retention [Box 1] and which extend beyond the constitutive donor splice site of exon 6 into the adjacent intron [Box 2]. All 4 reads which extend over the exon splice junction are derived from the G-allele (strong binding site; not all visible in panel $\mathbf{D})$. 

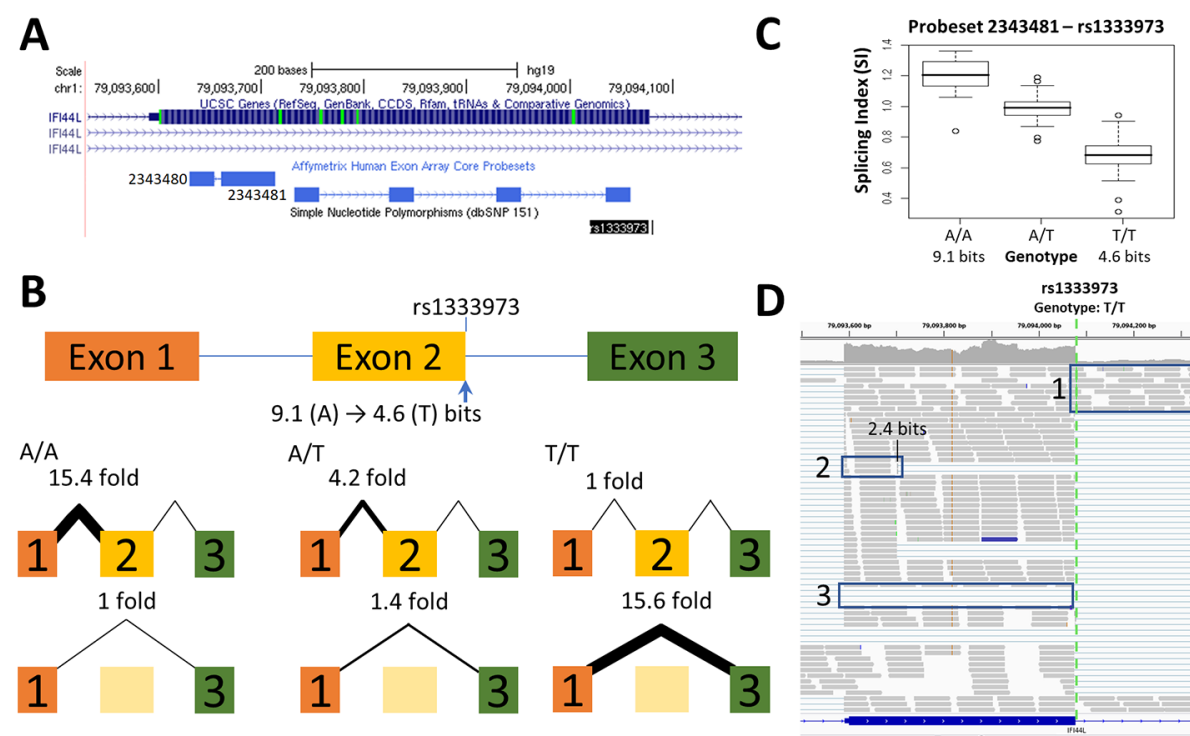

D

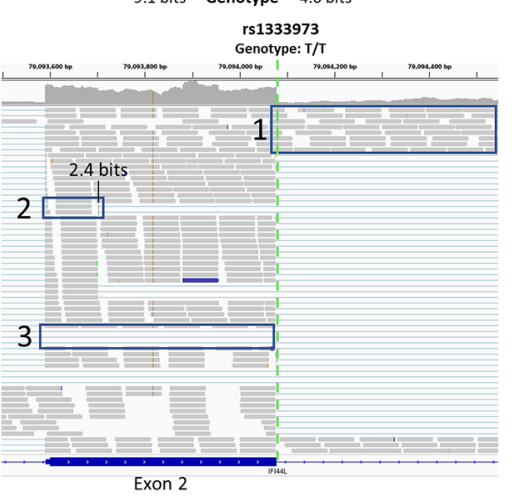

FIGURE 3 | Splicing Impact of $r 1333973$ (IF/44L). (A) The natural donor of IFI44L exon 2 is weakened from 9.1 to 4.6 bits (A> T) by rs1333973, increasing the frequency of exon 2 skipping and other events. (B) By q-RT-PCR, skipping was found to be 15.6-fold higher while normal splicing was 15.4-fold lower in A homozygotes (relative to T homozygotes). (C) Exon microarray data strongly supports the q-RT-PCR findings. (D) ICGC patient DO6354 is homozygous for the T-allele, which resulted in exon 2 skipping [Box 3], failure to recognize the exon 2 donor causing total retention of intron 2 [Box 1], and activation of an upstream exonic 2.4 bit cryptic donor 375 nt from the affected site [Box 2].

were found to reduce expression by 38 - to 58 -fold, the variance falling within the margin of measurement error. Modest natural splice site affinity changes predicted to be $<$ eightfold $\left(\Delta \mathrm{R}_{\mathrm{i}}<3.0\right)$ did not consistently result in detectable changes in splicing. In some instances, lower abundance splice forms were observed (i.e. rs2835585 altered exon skipping levels by up to 8.8 -fold; nevertheless, the normal splice form predominated).

\section{SNPs Affecting Cryptic Site Strength and Activity}

Increased cryptic site use coinciding with a decrease in natural site strength (Table 1) was validated for: rs1805377 (Figure 1); rs2243187 (Supplementary Image 1.4); rs3747107 (Supplementary Image 1.8); rs17002806 (Supplementary Image 1.13); rs6003906 (Supplementary Image 1.15); and rs13076750 (Supplementary Image 1.12). rs2070573 (Figure 2) and rs743920 (Supplementary Image 1.7) strengthened cryptic splice sites resulting in increased use of these sites. Despite the difference in strength between the natural and cryptic sites affected by rs743920, the upstream 2.4 bit site was used more frequently (Table 2). Both IL19 and XRCC4 regions tested showed preference to the upstream acceptor as well, which is consistent with the processive mechanism documented to recognize acceptor splice sites (Robberson et al., 1990).

\section{SNPs Affecting Exon Inclusion}

SNPs that reduced natural site strength $\left(\Delta R_{i}\right.$ from 1.6 to 10.9 bits) increased exon skipping from 2- to 27 -fold for homozygotes of differing genotypes of rs2835585 (Supplementary Image 1.21), rs1018448 (Supplementary Image 1.3), rs1333973 (Figure 3), rs2266988 (Supplementary Image 1.9), and rs13076750 (Supplementary Image 1.12). The exon microarray probesets for rs1018448 and rs1333973 detect decreased expression by genotype, which is consistent with increased exon skipping. Changes of average SI values did not correspond as well to specific genotypes for rs2835585 (TTC3), rs2266988 (PRAME), and rs13076750 (LPP), possibly due to increased cryptic site use $(L P P)$ or large differences in the abundance of constitutive and skipped isoforms (PRAME, TTC3).

\section{SNPs Promoting Alternate Exon Use}

SNP-related decreases in natural splice site strength may promote the use of alternative exons up or downstream of the affected exon. rs10190751 (Supplementary Image 1.2) is known to modulate the presence of the shorter c-FLIP(S) splice form of CFLAR (Ueffing et al., 2009). The use of this exon differed by $2^{17}$ fold between the strong and weak homozygotes tested, which was reflected by the expression microarray result. By q-RT-PCR, the CFLAR (L) form using an alternate downstream exon was found to be 2.1-fold more abundant in the homozygote with the weaker splice site. rs3747107 (Supplementary Image 1.8) and rs2285141 (Supplementary Image 1.20) exhibit evidence of an increased preference by q-RT-PCR for activation of an alternate exon, though the microarray results for the corresponding genotypes for both SNPs were not significantly different. 


\begin{tabular}{|c|c|c|c|c|c|c|c|c|c|c|c|c|}
\hline Gene & $\begin{array}{l}\text { Supplementary Image } 1 \text { panel / } \\
\text { rsID / HGVS notation }\end{array}$ & $\begin{array}{l}\text { Splice } \\
\text { type }\end{array}$ & $\begin{array}{c}\text { Information Change } \\
\text { (n-natural, c-cryptic site) }\end{array}$ & $\begin{array}{l}\text { Fold } \\
\text { change }\end{array}$ & $\begin{array}{l}\text { Natural } \\
\text { site }\end{array}$ & $\begin{array}{l}\text { Cryptic } \\
\text { site }\end{array}$ & $\begin{array}{l}\text { Exon } \\
\text { skipping }\end{array}$ & $\begin{array}{l}\text { Alternate } \\
\text { exon }\end{array}$ & $\begin{array}{l}\text { Total } \\
\text { mRNA }\end{array}$ & In-frame ${ }^{a}$ & $\begin{array}{l}\text { Exon } \\
\text { microarray }\end{array}$ & $\begin{array}{c}\text { RNAseq } \\
\text { (Valid-SpliceMut) }\end{array}$ \\
\hline XRCC4 & 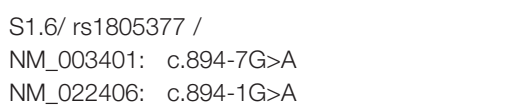 & A & $\begin{array}{c}11.5(G)->0.6(A)(n) \\
11.4(G)->11.8(A)(n)\end{array}$ & $\begin{array}{c}1886 \\
1.3\end{array}$ & $\begin{array}{l}38.4 \\
-\end{array}$ & $\begin{array}{c}- \\
47.3^{\mathrm{c}}\end{array}$ & $\begin{array}{l}- \\
-\end{array}$ & - & $\begin{array}{l}n / a^{b} \\
n / a^{b}\end{array}$ & $\begin{array}{l}Y \\
Y\end{array}$ & $\begin{array}{l}\mathrm{N} \\
\mathrm{N}\end{array}$ & $\begin{array}{l}Y \\
Y\end{array}$ \\
\hline IL19 & S1.4/ rs2243187 / NM_013371: c.364-1G>A & A & $4.7(G)->-6.2(A)(n)$ & 26.3 & $1.8^{\mathrm{d}}$ & $1.8^{\mathrm{d}}$ & $2.1^{\mathrm{d}}$ & - & $n / a^{b}$ & Y & Y & N \\
\hline C21orf2 & S1.1/ rs2070573 / NM_004928: c.643-137A>C & D & $0.4(\mathrm{~A})->4.0(\mathrm{C})(\mathrm{C})$ & 12.1 & NC & 22.6 & - & - & $n / a^{b}$ & Y & Y & Y \\
\hline$\pi$ псз & S1.21/rs2835655 / NM_003316: c.5115G>A & D & $11.2(\mathrm{G})->8.2(\mathrm{~A})(\mathrm{n})$ & 8.1 & 1.5 & - & $1.3^{\mathrm{e}}$ & - & 1.5 & - & $\mathrm{N}$ & $\mathrm{N}$ \\
\hline$\pi C 3^{f}$ & S1.5/rs2835585 / NM_003316: c.188-8T>A & A & $7.6(\mathrm{~T}) \rightarrow 5.4(\mathrm{~A})(\mathrm{n})$ & 4.5 & NC & - & 8.8 & - & $n / a^{b}$ & Y & $\mathrm{N}$ & Y \\
\hline WBP2NL & S1.13/rs17002806 / NM_152613: c. ${ }^{\star} 86 G>A$ & D & $9.2(\mathrm{G})->6.0(\mathrm{~A})(\mathrm{n})$ & 9.1 & 23.8 & $34+^{9}$ & - & - & $n / a^{b}$ & $\mathrm{~N}$ & Y & N \\
\hline GUSBP11 & $\begin{array}{l}\text { S1.8/ rs3747107 / NR_024448: n.2961+6512 } \\
\text { C>G }\end{array}$ & A & $4.7(\mathrm{C})->-7.0(\mathrm{G})(\mathrm{n})$ & 25.9 & 98.3 & $\begin{array}{c}31 / \\
42.8^{h}\end{array}$ & - & 1.6 & $n / a^{b}$ & $\mathrm{Y} / \mathrm{N}^{\mathrm{i}}$ & $\mathrm{N}$ & N \\
\hline PRAME & S1.9/ rs2266988k / NM_006115: c.19G>A & D & $7.8(\mathrm{G})->6.2(\mathrm{~A})(\mathrm{n})$ & 3.0 & NC & - & 8.8 & - & $n / a^{b}$ & $\mathrm{~N}$ & $\mathrm{~N}$ & N \\
\hline PRAME & S1.14/ rs2072049 / NM_006115: c.954-16G>T & A & $8.2(\mathrm{G})->7.1(\mathrm{~T})(\mathrm{n})$ & 2.2 & $2.6^{\mathrm{d}}$ & - & - & - & $3.1^{d}$ & - & Y & Y \\
\hline UBASHЗA & $\begin{array}{l}\text { S1.10/rs1893592 / } \\
\text { NM_018961: c.1393+3A>C }\end{array}$ & D & $8.7(\mathrm{~A})->4.2(\mathrm{C})(\mathrm{n})$ & 22.9 & 3.0 & $2.0 / 1.7^{\prime}$ & $N / A^{m}$ & - & 1.4 & Y & $\mathrm{N}$ & Y \\
\hline DERL3 & S1.15/rs6003906 / NM_198440: c.328-8A>T & A & $-2.1(A)->-4.3(T)(n)$ & 4.6 & NC & 2.0 & - & - & $n / a^{b}$ & $\mathrm{~N}$ & Y & N \\
\hline ARFGAP3 & S1.3/ rs1018448 / NM_014570: c.1065C>A & A & $10.5(\mathrm{C})->8.3(\mathrm{~A})(\mathrm{n})$ & 4.5 & 2.2 & - & 1.4 & - & 2.0 & $\mathrm{Y}$ & $\mathrm{Y}$ & $\mathrm{N}$ \\
\hline CFLAR & $\begin{array}{l}\text { S1.2/rs10190751 / NM_003879: c.606 } \\
\text { +934G>A NM_001127184:c.607-1G>A }\end{array}$ & A & $16.1(\mathrm{G})->5.2(\mathrm{~A})(\mathrm{n})$ & 1885 & $10000+$ & - & - & 2.1 & $n / a^{b}$ & Y & Y & Y \\
\hline IFI44L & S1.11/ rs1333973 / NM_006820: c.478+3A>T & D & $9.1(\mathrm{~A})->4.6(T)(n)$ & 22.6 & 15.4 & - & 15.6 & - & 3.9 & $\mathrm{~N}$ & Y & N \\
\hline LPP & $\begin{array}{l}\text { S1.12/ rs13076750 / NM_001167671:c.-66- } \\
\text { 8G>A }\end{array}$ & A & $9.3(\mathrm{G})->-1.6(\mathrm{~A})(\mathrm{n})$ & 625 & $5000+$ & 15.8 & 26.6 & - & - & Y & $\mathrm{N}$ & Y \\
\hline EMID1 & S1.7/ rs743920 / NM_133455: c.326C>G & A & $6.0(\mathrm{C})->7.9$ (G) (c) & 3.6 & $n / a^{b}$ & 5.8 & - & - & $n / a^{b}$ & - & Y & N \\
\hline CLDN14 & $\begin{array}{l}\text { S1.22/ rs16994182 / NM_001146078:C.-82 } \\
+4 C>G\end{array}$ & D & $7.4(\mathrm{C})->6.8(\mathrm{G})(\mathrm{n})$ & 1.6 & 5.9 & - & 2.1 & - & $n / a^{b}$ & Y & $\mathrm{N} / \mathrm{A}$ & N \\
\hline$B C R$ & S1.17/ rs16802 / NM_004327:c.2708-13A>G & A & $5.6(\mathrm{~A})->5.8(\mathrm{G})(\mathrm{n})$ & 1.1 & $N C^{n}$ & - & - & - & $n / a^{b}$ & - & $\mathrm{N}$ & N \\
\hline TMPRSS3 & S1.19 / rs8130564 / NM_032404: c.66-13T>C & A & $4.2(\mathrm{~T})->4.4(\mathrm{C})(\mathrm{n})$ & 1.1 & $N C^{n}$ & - & - & - & $n / a^{b}$ & - & $\mathrm{N}$ & N \\
\hline BACE2 & S1.18/ rs2252576 / NM_012105: c.748-10C>T & A & $7.2(\mathrm{C})->8.0(\mathrm{~T})(\mathrm{n})$ & 1.5 & $N C^{n}$ & - & - & - & $n / a^{b}$ & - & $\mathrm{N}$ & N \\
\hline CYB5R3 & S1.20/rs2285141 / NM_007326: c.-48-18G>T & A & $2.0(G)->1.2(T)(n)$ & 1.7 & NC & - & - & 1.8 & $n / a^{b}$ & - & $\mathrm{N}$ & N \\
\hline FАMЗВ & S1.16/rs2838010 / NM_058186: c.20-217A>T & D & $-10.8(\mathrm{~A})->7.8(\mathrm{~T})(\mathrm{C})$ & 228 & - & - & - & $N / A^{i}$ & $n / a^{b}$ & Y & $\mathrm{N}$ & $\mathrm{N}$ \\
\hline
\end{tabular}

Red text indicates a decrease in the abundance of a particular splice form, while green text indicates an increase in abundance. A - Acceptor Splice Site Affected; D-Donor Splice Site Affected; NC - Not detectable (abolished). ${ }^{2}$ Splicing events which alter reading frame may induce nonsense-mediated decay; ${ }^{b}$ No allele specific difference in expression and splicing; ${ }^{c}$ complete discrimination of both isoforms using a custom designed TaqMan probe; ${ }^{d}$ Values from comparing heterozygote with homozygote common; ${ }^{\circledR}$ Change in splicing likely related to change in RNA level; ${ }^{f}$ Intron 2-3 retention of TTC3 amplified by PCR, but no allele specific change detected; ${ }^{g}$ This splice form not at detectable levels in

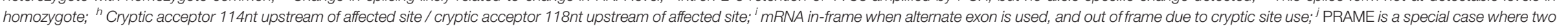

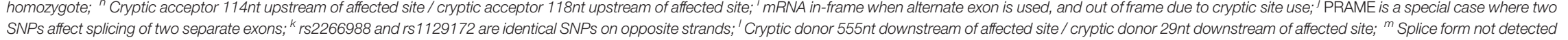
by PCR; ${ }^{n}$ High variability between individuals with the same genotype by $q-R T-P C R$. 
TABLE 2 | Abundance of mRNA splice forms relative to internal gene reference.

\begin{tabular}{|c|c|c|c|c|}
\hline Gene & rsID & mRNA Splice Form & $\begin{array}{c}\text { Homozygotes strong } \\
\text { allele (\%) } \\
\text { [\# Patients Tested] }\end{array}$ & $\begin{array}{c}\text { Homozygotes weak } \\
\text { allele (\%) } \\
\text { [\# Patients Tested] }\end{array}$ \\
\hline C21orf2 & rs2070573 & Extended Exon 6 Splice Form & $45.3 \pm 16.9[2]^{1}$ & $1.5 \pm 0.2[2]$ \\
\hline ТТСЗ & rs2835585 & Exon 3 Skipping & $<0.1 \pm 0.0[2]$ & $0.3 \pm 0.1[2]$ \\
\hline $1 L 19^{2}$ & rs2243187 & $3 \mathrm{nt}$ Inclusion of Exon 5 & $\sim 100[1]$ & $53.2 \pm 3.0$ [1; Het.] \\
\hline $1 L 19^{2}$ & rs2243187 & 3 nt Exclusion of Exon 5 & $61.0 \pm 3.4[1]$ & $\sim 100$ [1; Het.] \\
\hline $1 L 19^{2}$ & rs2243187 & Exon 5 Skipping & $14.1 \pm 1.4[1]$ & $6.8 \pm 1.1[1 ;$ Het. $]$ \\
\hline UBASHЗA & rs1893592 & 29 nt Retention of Intron 10 & $8.6 \pm 4.6[1]$ & $4.2 \pm 5.8[1]$ \\
\hline XRCC4 & rs1805377 & 6 nt Inclusion of Exon 8 & $\sim 100[1]$ & $3.1 \pm 0.5[1]$ \\
\hline XRCC4 & rs1805377 & 6 nt Exclusion of Exon 8 & $2.6 \pm 1.1[1]$ & $61.4 \pm 26.3[1]$ \\
\hline PRAME & rs2266988 & Normal Exon 3 Splicing & $32.4 \pm 3.6[1]$ & $20.1 \pm 3.9[1]$ \\
\hline PRAME & rs2266988 & Exon 3 Skipping & $1.2 \pm 1.2[1]$ & $3.5 \pm 3.0[1]$ \\
\hline GUSBP11 & rs3747107 & Exon 8 Splicing & $34.0 \pm 0.6[1]$ & $0.3 \pm 0.0[2]$ \\
\hline GUSBP11 & rs3747107 & Alternative Exon 8 & $37.3 \pm 7.3[1]$ & $39.8 \pm 3.7[2]$ \\
\hline GUSBP11 & rs3747107 & 114 nt Retention of Intron 7 & $0.1 \pm 0.0[1]$ & $1.4 \pm 0.1[2]$ \\
\hline GUSBP11 & rs3747107 & 118 nt Retention of Intron 7 & $0.2 \pm 0.0[1]$ & $8.0 \pm 0.5[2]$ \\
\hline DERL3 & rs6003906 & Normal Exon 5 Splicing & $\sim 100[1]$ & $55.8 \pm 0.0[1]$ \\
\hline DERL3 & rs6003906 & Extended Exon 4; Short Exon 5 & $3.2 \pm 0.0[1]$ & $4.0 \pm 0.0[1]$ \\
\hline ARFGAP3 & rs1018448 & Exon 12 Skipping & $12.3 \pm 5.7[3]$ & $23.1 \pm 10.1[1]$ \\
\hline IFI44L & rs1333973 & Normal Exon 2 Splicing & $57.0 \pm 0.0[1]$ & $14.7 \pm 0.0[1]$ \\
\hline IFI44L & rs1333973 & Exon 2 Skipping & $0.8 \pm 0.0[1]$ & $48.0 \pm 0.0[1]$ \\
\hline CFLAR & rs10190751 & Upstream Exon 7 Use & $\sim 100[1]$ & $<0.1 \pm 0.0[1]$ \\
\hline CFLAR & rs10190751 & Downstream Exon 7 Use & $39.0 \pm 0.0[1]$ & $87.1 \pm 0.0[1]$ \\
\hline WBP2NL & rs17002806 & 25 nt Intron 6 Retention & N.D. ${ }^{2}$ & $2.3 \pm 0.0[1]$ \\
\hline CYB5R3 & rs2285141 & Alternate Exon 2 Use & $<0.1 \pm 0.0[1]$ & $<0.1 \pm 0.0[1]$ \\
\hline EMID1 & rs743920 & $6 \mathrm{nt}$ deletion of Exon 4 & $69.0 \pm 9.7[1]$ & $9.2 \pm 1.4[1]$ \\
\hline CLDN14 & rs16994182 & Exon 2 Skipping & $1.9 \pm 0.0[1]$ & $5.3 \pm 0.0[1]$ \\
\hline
\end{tabular}

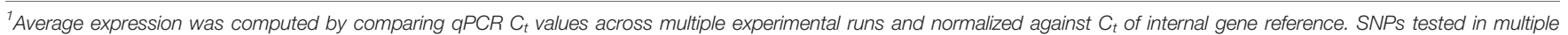

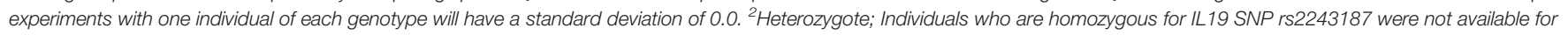
testing. N.D., Not detected. $C_{t}$ values were not available for LPP rs 13076750.

\section{SNP-Directed Effects on mRNA Levels}

A change in the strength of a natural site of an exon can affect the quantity of the processed mRNA (Caminsky et al., 2014). This decrease in mRNA could be caused by nonsense mediated decay (NMD), which degrades aberrant transcripts that would result in premature protein truncation (Cartegni et al., 2002). Of the 22 SNPs tested, 2 showed a direct correlation between a decrease in natural splice site strength, reduced amplification of the internal reference by q-RT-PCR (of multiple individuals) and a decreasing trend in expression by genotype by microarray: rs2072049 (Supplementary Image 1.9) and rs1018448 (Supplementary Image 1.3), although these differences do not meet statistical significance.

\section{SNPs With Pertinent Splicing Effects Detected by RNAseq}

Evidence for impact on splicing of the previously described SNPs was also assessed in TCGA and ICGC tumors by high throughput expression analyses. Splicing effects of these variants detected by q-RT-PCR and RNAseq were concordant in $80 \%$ of cases ( $\mathrm{N}=16$ of 20 SNPs), while impacts of $10 \%$ of SNPs $(\mathrm{N}=2)$ were partially concordant as a result of inconsistent activation of cryptic splice sites (Supplementary Images 1.8D and 1.10D). Several isoforms predicted by information analysis of these SNPs were present in complete transcriptomes, but were undetectable by q-RT-PCR or expression microarrays. Examples include a 4.9 bit cryptic site activated by rs3747107 located 2 nucleotides from the natural splice site (Supplementary Image 1.8D), exon skipping by rs1893592 (Supplementary Image 1.10D), and a cryptic exon activated by rs2838010 (Supplementary Image $\mathbf{1 . 1 6 C}$ ). Processed mRNAs that were not detected by q-RT-PCR may have arisen as a result of a lack of sensitivity of the assay, to NMD (which could mask detection of mis-splicing), to a deficiency of an undefined trans-acting splicing factor, or to design limitations in the experimental design. Another possibility is that the discordant splicing patterns of these two SNPs based could potentially be related to differences in tissue origin, since only the RNAseq findings were tumor-derived, whereas results obtained by the other approaches were generated from RNA extracted from lymphoblastoid cell lines. Cell culture conditions such as cell density and phosphorylation status can affect alternative splicing patterns (Li et al., 2006; Szafranski et al., 2014). These conditions, however, have not been studied in cases of allele-specific, sequence differences at splice sites or cis-acting regulatory sites that impact splice site selection. Considering the high level of concordance of splicing effects for the same SNPs in uncultured and cultured cells, it seems unlikely that culture conditions significantly impacts the majority of allele-specific, alternatively spliced isoforms. Our information theory-based analyses show that the dominant effect of SNP genotypes is to dictate common changes in splice site strength regardless of cell origin.

The results obtained from q-RT-PCR and RNAseq data for rs2070573, rs10190751, rs13076750, rs2072049, rs2835585, 
rs1893592, and rs1805377 were complementary to findings based on RNAseq (Supplementary Table 2). RNAseq data can reveal potential allele-specific alternate splicing events that were not considered at the primer design phase of the study, while qRT-PCR is more sensitive and can reveal less abundant alternative splice forms. A weak 0.4 bit splice site associated with rs2070573 was less abundant than the extended isoform (Figure 2) by both q-RT-PCR and exon microarray, however ValidSpliceMut also revealed increased total C21orf2 intron 6 retention in five tumors with this allele. Similarly, rs10190751 was flagged for intron retention in 29 tumors, which was not evident by the other approaches. The long form of this transcript (c-FLIP [L]) in homozygous carriers of this SNP was twice as abundant by q-RT-PCR than the shorter allele, associated with the weak splice site. rs13076750 activates an alternate acceptor site for a rare exon that extends the original exon length by seven nucleotides. The exon boundary can also extend into an adjacent exon, based on RNAseq of eight tumors carrying this SNP. Expression was decreased in the presence of a 6.2 bit splice site derived from a rs2072049 allele that weakens the natural acceptor site of the terminal exon of PRAME. The actual cause of diminished expression is likely to have been related to NMD from intron retention. ValidSpliceMut showed intron retention to be increased in rs2835585, whereas increased exon skipping for the allele with the weaker splice site was demonstrated by qRT-PCR. rs1893592 caused significant intron retention in all tumors $(\mathrm{N}=9)$, with exon skipping present in 3 diffuse large $B$ cell lymphoma patients, which was not detected by q-RT-PCR.
Finally, rs1805377 was associated with the significant abundance of read sequences indicating XRCC4 intron 7 retention by RNAseq $(\mathrm{N}=32)$, however this isoform could not be distinguished by the primers designed for q-RT-PCR and by TaqMan assay.

Alternative splicing events detected by RNAseq that were not evident in either q-RT-PCR or microarray studies included exon skipping induced by rs743920 (Supplementary Image 1.7D), activation of a preexisting cryptic splice site by rs1333973 (Figure 3), and intron retention by rs6003906 (Supplementary Image 1.15D). rs743920 creates an exonic hnRNP Al site $\left(R_{i}=\right.$ 2.8 bits) distant from the natural site which may compromise exon definition (Mucaki et al., 2013; Peterlongo et al., 2015) and may explain the SNP-associated increase in exon skipping. Exon definition analyses of total exon information $\left(R_{i, \text { total }}\right)$ also predicted the cryptic isoform arising from rs1333973 to be the most abundant $\left(R_{i, \text { total }}=9.4\right.$ bits $)$.

\section{Allele-Specific mRNA Splicing for Other SNPs Identified Through RNAseq}

A distinct set of 24 high population frequency SNPs were also evaluated for their potential impact on mRNA splicing by RNAseq analysis of ICGC patients. Those resulting in significantly decreased natural splice site strength $\left(\Delta R_{i}<-1\right.$ bit) were analyzed for SNP-derived alternative splicing events. SNPs fulfilling these criteria expressed at sufficient levels over the region of interest were: rs6467, rs36135, rs154290, rs166062, rs171632, rs232790, rs246391, rs324137, rs324726, rs448580,

TABLE 3 | RNAseq analysis of natural splice sites weakened by common single nucleotide polymorphisms (SNPS).

\begin{tabular}{|c|c|c|c|c|c|c|}
\hline Gene & rsID ${ }^{1}$ & $\begin{array}{l}\text { HGVS Notation } \\
\text { (HG19) }^{2}\end{array}$ & $\boldsymbol{R}_{i}$ initial & $R_{i}$ final & $\Delta R_{i}$ & Alternative Splicing Observed \\
\hline CYP21A2 & rs6467 & $6: 32006858 \mathrm{C}>\mathrm{A}$ & 6.1 & 4.5 & -1.6 & Intron Retention; Cryptic Site Use \\
\hline TRIM23 & rs36135 & $5: 64890479 A>C$ & 10.0 & 7.5 & -2.5 & Intron Retention \\
\hline ZFYE16 & rs166062 & $5: 79773028 \mathrm{~T}>\mathrm{G}$ & 14.1 & 11.8 & -2.4 & Intron Retention \\
\hline APBB3 & rs171632 & $5: 139941318 A>G$ & 2.5 & 1.4 & -1.1 & Intron Retention; Cryptic Site Use \\
\hline SMIM8 & rs448580 & 6:88040399T > G & 6.9 & 4.4 & -2.5 & Exon Skipping \\
\hline FCHSD1 & rs469074 & $5: 141024136 \mathrm{~T}>\mathrm{G}$ & 10.4 & 7.1 & -3.3 & Intron Retention \\
\hline KIFAP3 & rs518928 & $1: 169890933 A>G$ & 15.6 & 14.5 & -1.1 & Intron Retention \\
\hline CEPT1 & rs694180 & $1: 111726213 A>G$ & 9.6 & 7.0 & -2.6 & Intron Retention \\
\hline$A D C Y 10 P 1$ & rs722442 & $6: 41089681 A>G$ & 7.5 & 4.9 & -2.5 & Exon Skipping \\
\hline MICAL1 & rs752262 & $6: 109770999 G>C$ & 4.0 & 2.7 & -1.4 & Intron Retention \\
\hline MAP3K1 & rs832567 & $5: 56152416 C>A$ & 6.8 & 5.0 & -1.7 & Intron Retention; Exon Skipping \\
\hline METTL13 & rs909958 & $1: 171763522 \mathrm{C}>\mathrm{A}$ & 11.4 & 9.9 & -1.4 & Intron Retention \\
\hline DDX39B & rs933208 & $6: 31506648 G>T$ & 4.8 & 3.7 & -1.1 & Intron Retention; Cryptic Site Use \\
\hline CCT7 & rs1018342 & $2: 73471653 T>G$ & 5.9 & 4.4 & -1.5 & Intron Retention \\
\hline PPIP5K2 & rs154290 & $5: 102537200 T>G$ & 12.5 & 11.3 & -1.3 & Wildtype Only \\
\hline MYSM1 & rs232790 & $1: 59131311 G>T$ & 10.4 & 8.7 & -1.7 & Wildtype Only \\
\hline PDGFRB & rs246391 & $5: 149497177 \top>C$ & 6.2 & 3.6 & -2.6 & Wildtype Only \\
\hline AARS2 & rs324137 & $6: 44273546 A>C$ & 10.2 & 8.9 & -1.3 & Wildtype Only \\
\hline USO1 & rs324726 & $4: 76722353 G>A$ & 11.8 & 8.8 & -3.0 & Wildtype Only \\
\hline KIF13A & rs624105 & $6: 17855864 G>C$ & 14.1 & 13.0 & -1.1 & Wildtype Only \\
\hline TNFRSF1B & rs653667 & $1: 12251808 T>G$ & 3.7 & 2.4 & -1.3 & Wildtype Only \\
\hline CCDC93 & rs748767 & $2: 118731573 G>A$ & 4.6 & 3.4 & -1.1 & Wildtype Only \\
\hline CAPN2 & rs751128 & $1: 223951841 \top>C$ & 5.3 & 4.2 & -1.1 & Wildtype Only \\
\hline FYCO1 & rs751552 & $3: 46016851 \mathrm{~A}>\mathrm{T}$ & 6.9 & 4.7 & -2.2 & Wildtype Only \\
\hline
\end{tabular}

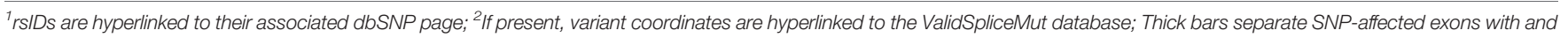
without RNAseq-observed alternate splicing events. 
rs469074, rs518928, rs624105, rs653667, rs694180, rs722442, rs748767, rs751128, rs751552, rs752262, rs832567, rs909958, rs933208, and rs1018342 (Table 3). Splicing was predicted to be leaky for all natural splice sites affected by these SNPs (Rogan et al., 1998; $R_{i, \text { final }} \geq 1.6$ bits), where reduction in $R_{i}$ values ranged from 1.1 to 3.3 bits.

Alternative mRNA splicing was observed in 14 SNPs: rs6467, rs36135, rs166062, rs171632, rs448580, rs469074, rs518928, rs694180, rs722442, rs752262, rs832567, rs909958, rs933208, rs1018342; Table 3). Reads spanning these regions revealed intron retention $(\mathrm{N}=12)$, activation of cryptic splicing $(\mathrm{N}=4)$, and complete exon skipping $(\mathrm{N}=3)$. Eleven of these SNPs (79\%) exhibited splicing patterns that significantly differed from the control alleles, and were therefore present in ValidSpliceMut. Interestingly, ValidSpliceMut contained entries for 7 of 10 SNPs where alternative splicing had not been found in the two patients reported in Table 3 (rs246391, rs324137, rs624105, rs653667, rs748767, rs751128, rs751552). The observed significant splicing differences for these SNPs occurred in distinct tumor types, consistent with tissue-specific effects of these SNPs on splicing.

\section{Instances of Limited Corroboration of SNP-Related Predictions}

Anticipated effects of the SNPs on splicing were not always confirmed by expression studies. Aside from incomplete or incorrect predictions, both design and execution of these studies as well as uncharacterized tissue specific effects could provide an explanation for these discrepancies. Furthermore, these undetected splicing events may have been targeted for NMD, however expression was not compared with mRNA levels from cells cultured with an inhibitor of protein translation. Stronger preexisting cryptic sites were, in some instances, not recognized nor was isoform abundance changed. These include: rs1893592 (6.4 and 5.2 bit cryptic donor sites 29 and 555 nt downstream of the affected donor); rs17002806 (a 5.7 bit site 67 nt downstream of the natural site); rs3747107 [creates a 4.9 bit cryptic site 2 nt downstream (observed by RNAseq; Supplementary Image 1.8 D)]; and rs2835585 (5.8 and 5.9 bit cryptic sites 60 and $87 \mathrm{nt}$ upstream of the natural site). SNPs with modestly decreased natural site strength ( 0.2 to 4.5 bits) did not consistently result in exon skipping (for example, rs1893592, rs17002806, and rs2835655).

Six SNPs predicted to disrupt natural splice sites could not be confirmed. Splicing effects were not identified in the 4 SNPs where the information change was $<1$ bit $(<$ twofold). Genetic variability masked potential splicing effects of three of these SNPs, including rs16802, rs2252576, and rs8130564 (Table 1). PCR primer sets designed for COL6A2 exon 21 (affected by rs17357592) did not produce the expected amplicon. Interpreting the results for rs16994182 (CLDN14) was complicated by the lack of a suitable internal reference. As CLDN14 consists of three exons, any internal reference covering the affected second exon cannot parse whether differences in exon 2 expression were caused by the SNP or by general expression changes.
The T-allele rs2838010 was predicted to activate a donor splice site of a rare exon in IVS1 of FAM3B (GenBank Accession AJ409094). The cryptic pseudoexon was neither detected by RT-PCR nor expression microarray (Supplementary Image 1.16). Interestingly, this exon is expressed in a malignant lymphoma patient who is a carrier for this genotype [ICGC ID: DO27769; (Supplementary Image 1.16C)]. Although the Tallele is probably required to activate the pseudoexon, additional unknown splicing-related factors appear to be necessary.

\section{DISCUSSION}

Predicted SNP alleles that alter constitutive mRNA splicing are confirmed by expression data, and appear to be a common cause of alternative splicing. The preponderance of leaky splicing mutations and cryptic splice sites, which often produce both normal and mutant transcripts, is consistent with balancing selection (Nuzhdin et al., 2004) or possibly with mutant loci that contribute to multifactorial disease. Minor SNP alleles are often found in $>1 \%$ of populations (Janosíková et al., 2005). This would be consistent with a bias against finding mutations that abolish splice site recognition in dbSNP. Such mutations are more typical in rare Mendelian disorders (Rogan et al., 1998).

Exon-based expression microarrays and q-RT-PCR techniques were initially used to confirm the predicted impact of common and rare SNPs on splicing. Results were subsequently confirmed using RNAseq data for some of these SNPs (Dorman et al., 2014; Viner et al., 2014; Shirley et al., 2019). However, exon skipping due to rs1893592 was not consistently seen in all carriers. Although detected only in one type of tumor, this event may not be tissue specific, since five patients with the same genotype did not exhibit this isoform. Nevertheless, exon skipping was also observed in malignant lymphoma (Supplementary Image 1.10D). Intron retention in rs 1805377 carriers was evident in only $22 \%$ of tumors. Increased total intron retention may be due to failure to recognize exons due to overlapping strong splice sites (Vockley et al., 2000; Rogan et al., 2003).

The splicing impacts of several of these SNPs have also been implicated in other studies. rs10190751 modulates the FLICEinhibitory protein (c-FLIP) from its S-form to its R-form, with the latter having been linked to increased lymphoma risk (Ueffing et al., 2009). We observed the R-form to be twice as abundant for one of the rs10190751 alleles. Increased exon skipping attributed to rs1333973 has been reported in RNAseq analysis of IFI44L (Zhao et al., 2013a), which has been implicated in reduced antibody response to measles vaccine (Haralambieva et al., 2017). The splicing impact of XRCC4 rs1805377 has been noted previously (Nalla and Rogan, 2005). This SNP has been implicated with an increased risk of gastric cancer (Chiu et al., 2008), pancreatic cancer (Ding and Li, 2015) and glioma (Zhao et al., 2013b). Similarly, the potential impact of rs 1893592 in UBASH3A has been recognized (Kim et al., 2015) and is associated with arthritis (Liu et al., 2017) and type 1 diabetes (Ge and Concannon, 2018). Hiller et al. (2006) described the 3 nt deletion caused by rs2243187 in IL19 but did not report increased exon skipping. 
rs743920 was associated with change in EMID1 expression (Ge et al., 2005), however its splicing impact was not recognized. Conversely, studies linking TMPRSS3 variants to hearing loss did not report rs8130564 to be significant (Lee et al., 2013; Chung et al., 2014). Interestingly, rs2252576 (in which we did not find a splicing alteration) has been associated to Alzheimer's dementia in Down syndrome (Mok et al., 2014).

rs2835585 significantly increased exon skipping in TTC3, however normal expression levels at the affected exon junction were not significantly altered. This was most likely due to the large difference in abundance between the constitutive and skipped splice isoforms (Table 2). The skipped isoform does not disrupt the reading frame and the affected coding region has not been assigned to any known protein domain (Tsukahara et al., 1996; Suizu et al., 2009). It is unclear whether allele-specific, exon skipping in this instance would impact TTC3 protein function or activity.

Why are so few natural splice sites strengthened by SNP-induced information changes? Most such changes would be thought to be neutral mutations, which are ultimately lost by chance (Fisher, 1930). Those variants which are retained are more likely to confer a selective advantage (Li, 1967). Indeed, the minor allele in rs2266988, which strengthens a donor splice site by 1.6 bits at the 5 ' end of the open reading frame in PRAME and occurs in $25 \%$ of the overall population ( $\sim 50 \%$ in Europeans). Several instances of modest changes in splice site strength that would be expected to have little or no impact, in fact, alter the degree of exon skipping.

Allele frequency can significantly vary across different populations, which can be indicative of gene flow and migration of a population (Cavalli-Sforza and Bodmer, 1971) as well as, in the case of splicing variants, genetic load and fitness in a population (Rogan and Mucaki, 2011). The frequencies of several of the variants presented here are significantly different between ethnic and geographically defined populations (Tables $\mathbf{1}$ and 3 ). We examined allele frequencies of these variants in sub-populations in both HapMap and dbSNP version 153. For example, representation of the alleles of the XRCC4 variant rs1805377 (where its A-allele leads to a $6 \mathrm{nt}$ deletion of the gene's terminal exon) differs between Caucasians and Asians (for the G- and A-alleles, respectively). Different linkage disequilibrium patterns of this variant occur in Han Chinese $(\mathrm{CHB})$ and Utah residents with Northern and Western European ancestry (CEU) populations (Zhao et al., 2013b). Similar differences in SNP population frequency include EMID1 rs743920 (in HapMap: G-allele frequency is $47 \%$ in $\mathrm{CHB}$, but only $7 \%$ in CEU and 10\% in northern Swedish cohorts). This is consistent with dbSNP (version 153) where it is present in $72 \%$ in Vietnamese, but only $16 \%$ of a northern Sweden cohort). In BACE2, rs2252576, the T-allele is most prevalent at $84 \%$ in Yoruba in Ibadan, Nigeria populations (YRI), but only $8 \%$ in $\mathrm{CHB}$. In FCHSD1, rs469074 the frequency of the G-allele is $37 \%$ in YRI and $<1 \%$ in CHB. Some SNPs were exclusively present in a single population in the HapMap cohort (e.g. only the YRI population is polymorphic for IL19 rs2243187, WBP2NL rs17002806, DERL3 rs6003906, and CLDN14 rs16994182).

Because of their effects on mRNA splicing, these differences in allele frequency would be expected to alter the relative abundance of certain protein isoforms in these populations. We speculate about whether isoform-specific representation among populations influences disease predisposition, other common phenotypic differences, or whether they are neutral. We suggest that SNPs decreasing constitutive splicing while increasing mRNA isoforms which alter the reading frame would be more likely to result in a distinct phenotype. q-RT-PCR experiments confirmed five SNPs which increased the fraction of mRNA splice forms causing a frameshift (Table 1), three of which simultaneously decrease constitutive splicing by $\geq 10$-fold (WBP2NL rs17002806; GUSBP11 rs3747107; and IFI44L rs1333973). rs3747107 and rs17002806 are much more common in YRI populations in HapMap (rs3747107 G-allele is present in 64\% in YRI but only $23 \%$ in CEU; rs17002806 A-allele was not identified in any CHB or CEU individuals), while the A-allele of rs1333973 is much more common in $\mathrm{CHB}$ (76\%, compared to $31 \%$ and $35 \%$ in CEU and YRI populations, respectively). These common variants are likely to change the function of these proteins and may influence individual phenotypes. A somewhat comprehensive catalog of DNA polymorphisms with splicing effects-confined or with increased prevalence in specific ethnic or geographically identifiable groupscould be derived from combining ValidSpliceMut with populationspecific SNP databases. Aside from those phenotypes described earlier, genes implicated by GWAS or other analyses for specific disorders represent reasonable candidates for further detailed or replication studies aimed at identification of the risk alleles in these cohorts.

The extent to which SNP-related sequence variation accounts for the heterogeneity in mRNA transcript structures has been somewhat unappreciated, given the relatively high proportion of genes that exhibit tissuespecific alternative splicing (Pan et al., 2008; Wang et al., 2008; Baralle and Giudice, 2017). This and our previous study (Shirley et al., 2019) raise questions regarding the degree to which apparent alternative splicing is the result of genomic polymorphism rather than splicing regulation alone. Because much of the information required for splice site recognition resides within neighboring introns, it would be prudent to consider contributions from intronic and exonic polymorphism that produce structural mRNA variation, since these changes might be associated with disease or predisposition.

Individual information corresponds to a continuous molecular phenotypic measure that is well suited to the analysis of contributions of multiple, incompletely penetrant SNPs in different genes, as typically seen in genetically complex diseases (Cooper et al., 2013). Our protocol identifies low or nonpenetrant allele-specific alternative splicing events through bioinformatic analysis, and either qRT-PCR, exon microarrays or RNAseq data analysis. Allelespecific splicing can also be determined by full-length alternative isoform analysis of RNA [or FLAIR (Workman et al., 2019)]. Differentiated splice forms are associated with specific alleles in heterozygotes with exonic SNPs. However, combining genome-based information with FLAIR may enable identification of intronic SNPs influencing splicing and low abundance alternative splice forms, which might 
otherwise be missed by FLAIR.

Targeted splicing analysis generally reproduces the results of our multi- genome-wide surveys of sequence variations affecting mRNA splicing. As splicing mutations and their effects were often observed in multiple tumor types, the impact of these mutations may be pleiotropic. Some events were only detected in q-RT-PCR data and not by RNAseq (and vice versa), highlighting the complementarity of these techniques for splicing mutation analyses. Results of this study increase confidence that the publicly (https://ValidSpliceMut. cytognomix.com) and commercially (https://MutationForecaster. com) available resources for information-theory based variant analysis and validation can distinguish mutations contributing to aberrant molecular phenotypes from allele-specific alternative splicing.

\section{DATA AVAILABILITY STATEMENT}

All datasets generated for this study are included in the article/ Supplementary Material.

\section{ETHICS STATEMENT}

Controlled-access TCGA and ICGC sequence data was approved by NCBI at the US National Institutes of Health (dbGaP Project \#988: "Predicting common genetic variants that alter the splicing of human gene transcripts"; Approval Number \#13930-11; PI: PK Rogan) and by the International Cancer Genome Consortium (ICGC Project \#DACO-1056047; "Validation of mutations that alter gene expression").

\section{REFERENCES}

Ars, E., Kruyer, H., Morell, M., Pros, E., Serra, E., Ravella, A., et al. (2003). Recurrent mutations in the NF1 gene are common among neurofibromatosis type 1 patients. J. Med. Genet. 40 (6), e82. doi: 10.1136/jmg.40.6.e82

Baralle, F. E., and Giudice, J. (2017). Alternative splicing as a regulator of development and tissue identity. Nat. Rev. Mol. Cell Biol. 18, 437-451. doi: $10.1038 / \mathrm{nrm} .2017 .27$

Berget, S. M. (1995). Exon recognition in vertebrate splicing. J. Biol. Chem. 270 (6), 2411-2414. doi: 10.1074/jbc.270.6.2411

Bi, C., and Rogan, P. K. (2004). Bipartite pattern discovery by entropy minimization-based multiple local alignment. Nucleic Acids Res. 32 (17), 4979-4991. doi: 10.1093/nar/gkh825

Birney, E., Kumar, S., and Krainer, A. R. (1993). Analysis of the RNA-recognition motif and RS and RGG domains: conservation in metazoan pre-mRNA splicing factors. Nucleic Acids Res. 21 (25), 5803-5816. doi: 10.1093/nar/ 21.25.5803

Black, D. L. (2003). Mechanisms of alternative pre-messenger RNA splicing. Annu. Rev. Biochem. 72, 291-336. doi: 10.1146/annurev.biochem.72.121801.161720

Buratti, E., Baralle, M., and Baralle, F. E. (2006). Defective splicing, disease and therapy: searching for master checkpoints in exon definition. Nucleic Acids Res. 34 (12), 3494-3510. doi: 10.1093/nar/gkl498

Cáceres, J. F., Stamm, S., Helfman, D. M., and Krainer, A. R. (1994). Regulation of alternative splicing in vivo by overexpression of antagonistic splicing factors. Science 265 (5179), 1706-1709. doi: 10.1126/science.8085156

Caminsky, N., Mucaki, E. J., and Rogan, P. K. (2014). Interpretation of mRNA splicing mutations in genetic disease: review of the literature and guidelines for informationtheoretical analysis. F1000Res 3, 282. doi: 10.12688/f1000research.5654.1

\section{AUTHOR CONTRIBUTIONS}

EM designed and performed all q-RT-PCR experiments, processed and analyzed publicly exon microarray data, and performed all formal analysis. BS performed data curation and software development. PR conceptualized the project and was the project administrator. EM and PR prepared the original draft of the manuscript, while EM, BS, and PR reviewed and edited the document.

\section{ACKNOWLEDGMENTS}

PR acknowledges support from the Natural Sciences and Engineering Research Council of Canada (NSERC) [371758-09 and RGPIN-2015-06290], Canadian Foundation for Innovation, Canada Research Chairs, and CytoGnomix. Cell lines were obtained from the NIGMS Human Genetic Cell Repository under a Materials Transfer Agreement with the Coriel Institute (Camden, NJ). An earlier version of this article is available from bioRxiv: https://www.biorxiv.org/content/10.1101/549089v2 (Mucaki and Rogan, 2019).

\section{SUPPLEMENTARY MATERIAL}

The Supplementary Material for this article can be found online at: https://www.frontiersin.org/articles/10.3389/fgene.2020. 00109/full\#supplementary-material

Caminsky, N. G., Mucaki, E. J., Perri, A. M., Lu, R., Knoll, J. H., and Rogan, P. K. (2016). Prioritizing variants in complete hereditary breast and ovarian cancer genes in patients lacking known BRCA mutations. Hum. Mutat. 37 (7), 640652. doi: 10.1002/humu.22972

Carothers, A. M., Urlaub, G., Grunberger, D., and Chasin, L. A. (1993). Splicing mutants and their second-site suppressors at the dihydrofolate reductase locus in Chinese hamster ovary cells. Mol. Cell Biol. 13 (8), 5085-5098. doi: 10.1128/ MCB.13.8.5085

Cartegni, L., Chew, S. L., and Krainer, A. R. (2002). Listening to silence and understanding nonsense: exonic mutations that affect splicing. Nat. Rev. Genet. 3 (4), 285-298. doi: 10.1038/nrg775

Caux-Moncoutier, V., Pagès-Berhouet, S., Michaux, D., Asselain, B., Castéra, L., De Pauw, A., et al. (2009). Impact of BRCA1 and BRCA2 variants on splicing: clues from an allelic imbalance study. Eur. J. Hum. Genet. 17 (11), 1471-1480. doi: 10.1038/ejhg.2009.89

Cavalli-Sforza, L. L., and Bodmer, W. F. (1971). The Genetics of Human Populations (San Francisco: W.H. Freeman and Company).

Chiu, C. F., Wang, C. H., Wang, C. L., Lin, C. C., Hsu, N. Y., Weng, J. R., et al. (2008). A novel single nucleotide polymorphism in XRCC4 gene is associated with gastric cancer susceptibility in Taiwan. Ann. Surg. Oncol. 15 (2), 514-518. doi: 10.1245/s10434-007-9674-3

Chung, J., Park, S. M., Chang, S. O., Chung, T., Lee, K. Y., Kim, A. R., et al. (2014). A novel mutation of TMPRSS3 related to milder auditory phenotype in Korean postlingual deafness: a possible future implication for a personalized auditory rehabilitation. J. Mol. Med. (Berl) 92 (6), 651-663. doi: 10.1007/s00109-014-1128-3 Colombo, M., De Vecchi, G., Caleca, L., Foglia, C., Ripamonti, C. B., Ficarazzi, F., et al. (2013). Comparative in vitro and in silico analyses of variants in splicing 
regions of BRCA1 and BRCA2 genes and characterization of novel pathogenic mutations. PloS One 8 (2), e57173. doi: 10.1371/journal.pone.0057173

Cooper, D. N., Krawczak, M., Polychronakos, C., Tyler-Smith, C., and KehrerSawatzki, H. (2013). Where genotype is not predictive of phenotype: towards an understanding of the molecular basis of reduced penetrance in human inherited disease. Hum Genet. 132(10), 1077-1130. doi: 10.1007/s00439-013$1331-2$

Dietz, H. C., Valle, D., Francomano, C. A., Kendzior, R. J. Jr, Pyeritz, R. E., and Cutting, G. R. (1993). The skipping of constitutive exons in vivo induced by nonsense mutations. Science 259 (5095), 680-683. doi: 10.1126/ science. 8430317

Ding, Y., and Li, L. N. (2015). Association between single nucleotide polymorphisms of $\mathrm{X}$-ray repair cross-complementing protein 4 gene and development of pancreatic cancer. Genet. Mol. Res. 14 (3), 9626-9632. doi: 10.4238/2015.August.14.25

Domenjoud, L., Kister, L., Gallinaro, H., and Jacob, M. (1993). Selection between a natural and a cryptic 5' splice site: a kinetic study of the effect of upstream exon sequences. Gene Expr. 3 (1), 83-94.

Dorman, S. N., Viner, C., and Rogan, P. K. (2014). Splicing mutation analysis reveals previously unrecognized pathways in lymph node-invasive breast cancer. Sci. Rep. 4, 7063. doi: 10.1038/srep07063

Eliseos, E. J., Shirley, B. S., and Rogan, P. K. (2019). Expression changes confirm predicted single nucleotide variants affecting mRNA splicing. bioRxiv, 549089. doi: $10.1101 / 549089$

Fisher, R. A. (1930). The genetical theory of natural selection (Oxford New York: Clarendon Press).

Fu, X. D., and Maniatis, T. (1992). The 35-kDa mammalian splicing factor SC35 mediates specific interactions between $\mathrm{U} 1$ and $\mathrm{U} 2$ small nuclear ribonucleoprotein particles at the 3 ' splice site. Proc. Natl. Acad. Sci. U.S.A. 89 (5), 1725-1729. doi: 10.1073/pnas.89.5.1725

Ge, Y., and Concannon, P. (2018). Molecular-genetic characterization of common, noncoding UBASH3A variants associated with type 1 diabetes. Eur. J. Hum. Genet. 26 (7), 1060-1064. doi: 10.1038/s41431-018-0123-5

Ge, B., Gurd, S., Gaudin, T., Dore, C., Lepage, P., Harmsen, E., et al. (2005). Survey of allelic expression using EST mining. Genome Res. 15 (11), 1584-1591. doi: $10.1101 /$ gr.4023805

Giardine, B., Riemer, C., Hardison, R. C., Burhans, R., Elnitski, L., Shah, P., et al. (2005). Galaxy: a platform for interactive large-scale genome analysis. Genome Res. 15 (10), 1451-1455. doi: 10.1101/gr.4086505

Gloss, B. S., and Dinger, M. E. (2018). Realizing the significance of noncoding functionality in clinical genomics. Exp. Mol. Med. 50 (8), 97. doi: 10.1038/s12276-018-0087-0

Haralambieva, I. H., Ovsyannikova, I. G., Kennedy, R. B., Larrabee, B. R., Zimmermann, M. T., Grill, D. E., et al. (2017). Genome-wide associations of CD46 and IFI44L genetic variants with neutralizing antibody response to measles vaccine. Hum. Genet. 136 (4), 421-435. doi: 10.1007/s00439-017-1768-9

Hiller, M., Huse, K., Szafranski, K., Jahn, N., Hampe, J., Schreiber, S., et al. (2006). Single-nucleotide polymorphisms in NAGNAG acceptors are highly predictive for variations of alternative splicing. Am. J. Hum. Genet. 78 (2), 291-302. doi: $10.1086 / 500151$

Hobson, G. M., Huang, Z., Sperle, K., Sistermans, E., Rogan, P. K., Garbern, J. Y., et al. (2006). Splice-site contribution in alternative splicing of PLP1 and DM20: molecular studies in oligodendrocytes. Hum. Mutat. 27 (1), 69-77. doi: 10.1002/humu.20276

Huang, R. S., Duan, S., Bleibel, W. K., Kistner, E. O., Zhang, W., Clark, T. A., et al. (2007). A genome-wide approach to identify genetic variants that contribute to etoposide-induced cytotoxicity. Proc. Natl. Acad. Sci. U. S. A. 104 (23), 97589763. doi: 10.1073/pnas.0703736104

Hull, J., Campino, S., Rowlands, K., Chan, M. S., Copley, R. R., Taylor, M. S., et al. (2007). Identification of common genetic variation that modulates alternative splicing. PloS Genet. 3 (6), e99. doi: 10.1371/journal.pgen.0030099

Janosíková, B., Zavadáková, P., and Kozich, V. (2005). Single-nucleotide polymorphisms in genes relating to homocysteine metabolism: how applicable are public SNP databases to a typical European population? Eur. J. Hum. Genet. 13 (1), 86-95. doi: 10.1038/sj.ejhg.5201282

Kannabiran, C., Rogan, P. K., Olmos, L., Basti, S., Rao, G. N., Kaiser-Kupfer, M., et al. (1998). Autosomal dominant zonular cataract with sutural opacities is associated with a splice mutation in the betaA3/A1-crystallin gene. Mol. Vis. 4, 21.

Kent, W. J., Sugnet, C. W., Furey, T. S., Roskin, K. M., Pringle, T. H., Zahler, A. M., et al. (2002). The human genome browser at UCSC. Genome Res. 12(6), 9961006. doi: $10.1101 /$ gr. 229102
Khan, S. G., Metin, A., Gozukara, E., Inui, H., Shahlavi, T., Muniz-Medina, V., et al. (2004). Two essential splice lariat branchpoint sequences in one intron in a xeroderma pigmentosum DNA repair gene: mutations result in reduced XPC mRNA levels that correlate with cancer risk. Hum. Mol. Genet. 13 (3), 343-352. doi: 10.1093/hmg/ddh026

Kim, K., Bang, S. Y., Lee, H. S., Cho, S. K., Choi, C. B., Sung, Y. K., et al. (2015). High-density genotyping of immune loci in Koreans and Europeans identifies eight new rheumatoid arthritis risk loci. Ann. Rheumatol. Dis. 74 (3), e13. doi: 10.1136/annrheumdis-2013-204749

Krawczak, M., Reiss, J., and Cooper, D. N. (1992). The mutational spectrum of single base-pair substitutions in mRNA splice junctions of human genes: causes and consequences. Hum. Genet. 90 (1-2), 41-54. doi: 10.1007/BF00210743

López-Bigas, N., Audit, B., Ouzounis, C., Parra, G., and Guigó, R. (2005). Are splicing mutations the most frequent cause of hereditary disease? FEBS Lett. 579 (9), 1900-1903. doi: 10.1016/j.febslet.2005.02.047

Lacroix, M., Lacaze-Buzy, L., Furio, L., Tron, E., Valari, M., Van der Wier, G., et al. (2012). Clinical expression and new SPINK5 splicing defects in Netherton syndrome: unmasking a frequent founder synonymous mutation and unconventional intronic mutations. J. Invest. Dermatol. 132 (3 Pt 1), 575-582. doi: $10.1038 /$ jid.2011.366

Lamba, V., Lamba, J., Yasuda, K., Strom, S., Davila, J., Hancock, M. L., et al. (2003). Hepatic CYP2B6 expression: gender and ethnic differences and relationship to CYP2B6 genotype and CAR (constitutive androstane receptor) expression. J. Pharmacol. Exp. Ther. 307 (3), 906-922. doi: 10.1124/jpet.103.054866

Lee, J., Baek, J. I., Choi, J. Y., Kim, U. K., Lee, S. H., and Lee, K. Y. (2013). Genetic analysis of TMPRSS3 gene in the Korean population with autosomal recessive nonsyndromic hearing loss. Gene 532 (2), 276-280. doi: 10.1016/ j.gene.2013.07.108

Li, C., Kato, M., Shiue, L., Shively, J. E., Ares, M., and Lin, R. J. (2006). Cell type and culture condition-dependent alternative splicing in human breast cancer cells revealed by splicing-sensitive microarrays. Cancer Res. 66 (4), 1990-1999. doi: 10.1158/0008-5472.CAN-05-2593

Li, C. C. (1967). Genetic equilibrium under selection. Biometrics 23, 397-484. doi: $10.2307 / 2528009$

Liu, D., Liu, J., Cui, G., Yang, H., Cao, T., and Wang, L. (2017). Evaluation of the association of UBASH3A and SYNGR1 with rheumatoid arthritis and disease activity and severity in Han Chinese. Oncotarget 8 (61), 103385-103392. doi: 10.18632 /oncotarget.21875

Lu, R., and Rogan, P. K. (2019). Transcription factor binding site clusters identify target genes with similar tissue-wide expression and buffer against mutations [version 2; peer review: 2 approved]. F1000Research 7, 1933. doi: 10.12688/ f1000research.17363.2

Lu, R., Mucaki, E. J., and Rogan, P. K. (2017). Discovery and validation of information theory-based transcription factor and cofactor binding site motifs. Nucleic Acids Res. 45 (5), e27. doi: 10.1093/nar/gkw1036

Margulis, V., Lin, J., Yang, H., Wang, W., Wood, C. G., and Wu, X. (2008). Genetic susceptibility to renal cell carcinoma: the role of DNA double-strand break repair pathway. Cancer Epidemiol. Biomarkers Prev. 17 (9), 2366-2373. doi: 10.1158/1055-9965.EPI-08-0259

Martinez-Contreras, R., Cloutier, P., Shkreta, L., Fisette, J. F., Revil, T., and Chabot, B. (2007). hnRNP proteins and splicing control. Adv. Exp. Med. Biol. 623, 123147. doi: 10.1007/978-0-387-77374-2_8

Mayeda, A., and Krainer, A. R. (1992). Regulation of alternative pre-mRNA splicing by hnRNP A1 and splicing factor SF2. Cell 68 (2), 365-375. doi: 10.1016/0092-8674(92)90477-T

Mok, K. Y., Jones, E. L., Hanney, M., Harold, D., Sims, R., Williams, J., et al. (2014). Polymorphisms in BACE2 may affect the age of onset Alzheimer's dementia in Down syndrome. Neurobiol. Aging 35 (6), 1513.e1-5. doi: 10.1016/ j.neurobiolaging.2013.12.022

Moore, M. J., and Sharp, P. A. (1993). Evidence for two active sites in the spliceosome provided by stereochemistry of pre-mRNA splicing. Nature 365 (6444), 364-368. doi: 10.1038/365364a0

Mucaki, E. J., Shirley, B. C., and Rogan, P. K. (2013). Prediction of mutant mRNA splice isoforms by information theory-based exon definition. Hum. Mutat. 34 (4), 557-565. doi: 10.1002/humu.22277

Mucaki, E. J., Caminsky, N. G., Perri, A. M., Lu, R., Laederach, A., Halvorsen, M., et al. (2016). A unified analytic framework for prioritization of non-coding variants of uncertain significance in heritable breast and ovarian cancer. $B M C$ Med. Genomics 9, 19. doi: 10.1186/s12920-016-0178-5 
Nalla, V. K., and Rogan, P. K. (2005). Automated splicing mutation analysis by information theory. Hum. Mutat. 25 (4), 334-342. doi: 10.1002/humu.20151

Nelson, K. K., and Green, M. R. (1990). Mechanism for cryptic splice site activation during pre-mRNA splicing. Proc. Natl. Acad. Sci. U.S.A. 87 (16), 6253-6257. doi: 10.1073/pnas.87.16.6253

Nembaware, V., Lupindo, B., Schouest, K., Spillane, C., Scheffler, K., and Seoighe, C. (2008). Genome-wide survey of allele-specific splicing in humans. BMC Genomics 9, 265. doi: 10.1186/1471-2164-9-265

Nuzhdin, S. V., Wayne, M. L., Harmon, K. L., and McIntyre, L. M. (2004). Common pattern of evolution of gene expression level and protein sequence in Drosophila. Mol. Biol. Evol. 21 (7), 1308-1317. doi: 10.1093/molbev/msh128

Olsen, R. K. , Brøner, S., Sabaratnam, R. , Doktor, T. K. , Andersen, H. S., Bruun, G. H., et al. (2014). The ETFDH c.158A $>\mathrm{G}$ variation disrupts the balanced interplay of ESE- and ESS-binding proteins thereby causing missplicing and multiple Acyl-CoA dehydrogenation deficiency. Hum. Mutat. 35 (1), 86-95. doi: 10.1002/humu.22455

Pan, Q., Shai, O., Lee, L. J., Frey, B. J., and Blencowe, B. J. (2008). Deep surveying of alternative splicing complexity in the human transcriptome by highthroughput sequencing. Nat. Genet. 40, 1413-1415. doi: 10.1038/ng.259

Park, E., Pan, Z., Zhang, Z., Lin, L., and Xing, Y. (2018). The expanding landscape of alternative splicing variation in human populations. Am. J. Hum. Genet. 102 (1), 11-26. doi: 10.1016/j.ajhg.2017.11.002

Parker, R., Siliciano, P. G., and Guthrie, C. (1987). Recognition of the TACTAAC box during mRNA splicing in yeast involves base pairing to the U2-like snRNA. Cell 49 (2), 229-239. doi: 10.1016/0092-8674(87)90564-2

Peterlongo, P., Catucci, I., Colombo, M., Caleca, L., Mucaki, E., Bogliolo, M., et al. (2015). FANCM c.5791C > T nonsense mutation (rs144567652) induces exon skipping, affects DNA repair activity and is a familial breast cancer risk factor. Hum. Mol. Genet. 24 (18), 5345-5355. doi: 10.1093/hmg/ddv251

Pfaffl, M. W., Horgan, G. W., and Dempfle, L. (2002). Relative expression software tool (REST) for groupwise comparison and statistical analysis of relative expression results in real-time PCR. Nucleic Acids Res. 30 (9), e36. doi: 10.1093/nar/30.9.e36

Richard, I., and Beckmann, J. S. (1995). How neutral are synonymous codon mutations? Nat. Genet. 10 (3), 259. doi: 10.1038/ng0795-259

Robberson, B. L., Cote, G. J., and Berget, S. M. (1990). Exon definition may facilitate splice site selection in RNAs with multiple exons. Mol. Cell Biol. 10 (1), 84-94. doi: 10.1128/MCB.10.1.84

Robinson, J. T., Thorvaldsdóttir, H., Wenger, A. M., Zehir, A., and Mesirov, J. P. (2017). Variant Review with the Integrative Genomics Viewer. Cancer Res. 77 (21), e31-e34. doi: 10.1158/0008-5472.CAN-17-0337

Rogan, P. K., and Mucaki, E. J. (2011). Population fitness and genetic load of single nucleotide polymorphisms affecting mRNA splicing. arXiv:1107.0716 [q-bio.GN].

Rogan, P. K., and Schneider, T. D. (1995). Using information content and base frequencies to distinguish mutations from genetic polymorphisms in splice junction recognition sites. Hum. Mutat. 6 (1), 74-76. doi: 10.1002/humu.1380060114

Rogan, P. K., Faux, B. M., and Schneider, T. D. (1998). Information analysis of human splice site mutations. Hum. Mutat. 12, 153-171. doi: 10.1002/(SICI) 1098-1004(1998)12:3<153::AID-HUMU3>3.0.CO;2-I

Rogan, P. K., Svojanovsky, S. R., and Leeder, J. S. (2003). Information theory-based analysis of CYP219, CYP2D6 and CYP3A5 splicing mutations. Pharmacogenetics 13 (4), 207-218. doi: 10.1097/00008571-200304000-00005

Séraphin, B., Kretzner, L., and Rosbash, M. (1988). A U1 snRNA:pre-mRNA base pairing interaction is required early in yeast spliceosome assembly but does not uniquely define the 5 ' cleavage site. EMBO J. 7 (8), 2533-2538. doi: 10.1002/ j.1460-2075.1988.tb03101.x

Schneider, T. D. (1997). Sequence walkers: a graphical method to display how binding proteins interact with DNA or RNA sequences. Nucleic Acids Res. 25, 4408-4415. doi: 10.1093/nar/25.21.4408

Shirley, B. C., Mucaki, E. J., Whitehead, T., Costea, P. I., Akan, P., and Rogan, P. K. (2013). Interpretation, stratification and evidence for sequence variants affecting mRNA splicing in complete human genome sequences. Genomics Proteomics Bioinf. 11 (2), 77-85. doi: 10.1016/j.gpb.2013.01.008

Shirley, B. C., Mucaki, E. J., and Rogan, P. K. (2019). Pan-cancer repository of validated natural and cryptic mRNA splicing mutations [version 3; peer review: 1 approved, 2 approved with reservations]. F1000Research 7, 1908. doi: 10.12688/f1000research.17204.3

Soemedi, R., Cygan, K. J., Rhine, C. L., Wang, J., Bulacan, C., Yang, J., et al. (2017). Pathogenic variants that alter protein code often disrupt splicing. Nat. Genet. 49 (6), 848-855. doi: 10.1038/ng.3837
Spielmann, M., and Mundlos, S. (2016). Looking beyond the genes: the role of non-coding variants in human disease. Hum. Mol. Genet. 25 (R2), R157-R165. doi: $10.1093 / \mathrm{hmg} / \mathrm{ddw} 205$

Staknis, D., and Reed, R. (1994). SR proteins promote the first specific recognition of Pre-mRNA and are present together with the U1 small nuclear ribonucleoprotein particle in a general splicing enhancer complex. Mol. Cell Biol. 14 (11), 7670-7682. doi: 10.1128/MCB. 14.11 .7670

Suizu, F., Hiramuki, Y., Okumura, F., Matsuda, M., Okumura, A. J., Hirata, N., et al. (2009). The E3 ligase TTC3 facilitates ubiquitination and degradation of phosphorylated Akt. Dev. Cell. 17 (6), 800-810. doi: 10.1016/ j.devcel.2009.09.007

Susani, L., Pangrazio, A., Sobacchi, C., Taranta, A., Mortier, G., Savarirayan, R., et al. (2004). TCIRG1-dependent recessive osteopetrosis: mutation analysis, functional identification of the splicing defects, and in vitro rescue by $\mathrm{U} 1$ snRNA. Hum. Mutat. 24 (3), 225-235. doi: 10.1002/humu.20076

Svojanovsky, S. R., Schneider, T. D., and Rogan, P. K. (2000). Redundant designations of BRCA1 intron 11 splicing mutation; c. 4216-2A > G; IVS112A > G; L78833, 37698, A > G. Hum. Mutat. 16 (3), 264. doi: 10.1002/10981004(200009)16:3<264::AID-HUMU9>3.0.CO;2-1

Szafranski, K., Fritsch, C., Schumann, F., Siebel, L., Sinha, R., Hampe, J., et al. (2014). Physiological state co-regulates thousands of mammalian mRNA splicing events at tandem splice sites and alternative exons. Nucleic Acids Res. 42 (14), 8895-8904. doi: 10.1093/nar/gku532

Talerico, M., and Berget, S. M. (1990). Effect of 5' splice site mutations on splicing of the preceding intron. Mol. Cell Biol. 10 (12), 6299-6305. doi: 10.1128/ MCB.10.12.6299

Teraoka, S. N., Telatar, M., Becker-Catania, S., Liang, T., Onengüt, S., Tolun, A., et al. (1999). Splicing defects in the ataxia-telangiectasia gene, ATM: underlying mutations and consequences. Am. J. Hum. Genet. 64 (6), $1617-$ 1631. doi: $10.1086 / 302418$

Thorsen, K., Sørensen, K. D., Brems-Eskildsen, A. S., Modin, C., Gaustadnes, M., Hein, A. M., et al. (2008). Alternative splicing in colon, bladder, and prostate cancer identified by exon array analysis. Mol. Cell Proteomics 7 (7), 1214-1224. doi: 10.1074/mcp.M700590-MCP200

Tsukahara, F., Hattori, M., Muraki, T., and Sakaki, Y. (1996). Identification and cloning of a novel cDNA belonging to tetratricopeptide repeat gene family from Down syndrome-critical region 21q22.2. J. Biochem. 120 (4), 820-827. doi: 10.1093/oxfordjournals.jbchem.a021485

Ueffing, N., Singh, K. K., Christians, A., Thorns, C., Feller, A. C., Nagl, F., et al. (2009). A single nucleotide polymorphism determines protein isoform production of the human c-FLIP protein. Blood 114 (3), 572-579. doi: 10.1182/blood-2009-02-204230

Vemula, S. R., Xiao, J., Zhao, Y., Bastian, R. W., Perlmutter, J. S., Racette, B. A., et al. (2014). A rare sequence variant in intron 1 of THAP1 is associated with primary dystonia. Mol. Genet. Genom. Med. 2 (3), 261-272. doi: 10.1002/ mgg3.67

Viner, C., Dorman, S. N., Shirley, B. C., and Rogan, P. K. (2014). Validation of predicted mRNA splicing mutations using high-throughput transcriptome data [version 2; referees: 4 approved]. F1000Res 3, 8. doi: 10.12688/ f1000research.3-8.v2

Vockley, J., Rogan, P. K., Anderson, B. D., Willard, J., Seelan, R. S., Smith, D. I., et al. (2000). Exon skipping in IVD RNA processing in isovaleric acidemia caused by point mutations in the coding region of the IVD gene. Am. J. Hum. Genet. 66 (2), 356-367. doi: 10.1086/302751

von Kodolitsch, Y., Pyeritz, R. E., and Rogan, P. K. (1999). Splice-site mutations in atherosclerosis candidate genes: relating individual information to phenotype. Circulation 100 (7), 693-699. doi: 10.1161/01.CIR.100.7.693

von Kodolitsch, Y., Berger, J., and Rogan, P. K. (2006). Predicting severity of haemophilia A and B splicing mutations by information analysis. Haemophilia 12 (3), 258-262. doi: 10.1111/j.1365-2516.2006.01216.x

Vyhlidal, C. A., Rogan, P. K., and Leeder, J. S. (2004). Development and refinement of pregnane $\mathrm{X}$ receptor (PXR) DNA binding site model using information theory: insights into PXR-mediated gene regulation. J. Biol. Chem. 279 (45), 46779-46786. doi: 10.1074/jbc.M408395200

Wang, E. T., Sandberg, R., Luo, S., Khrebtukova, I., Zhang, L., Mayr, C., et al. (2008). Alternative isoform regulation in human tissue transcriptomes. Nature 456 (7221), 470-476. doi: 10.1038/nature07509 
Workman, R. E., Tang, A. D., Tang, P. S., Jain, M., Tyson, J. R., Razaghi, R., et al. (2019). Nanopore native RNA sequencing of a human poly(A) transcriptome. Nat. Methods. doi: 10.1038/s41592-019-0617-2

Wu, J., and Manley, J. L. (1989). Mammalian pre-mRNA branch site selection by U2 snRNP involves base pairing. Genes Dev. 3 (10), 1553-1561. doi: 10.1101/ gad.3.10.1553

Wu, X., Gu, J., Grossman, H. B., Amos, C. I., Etzel, C., Huang, M., et al. (2006). Bladder cancer predisposition: a multigenic approach to DNA-repair and cell-cycle-control genes. Am. J. Hum. Genet. 78 (3), 464-479. doi: 10.1086/500848

Yates, T., Okoniewski, M. J., and Miller, C. J. (2008). X:Map: annotation and visualization of genome structure for Affymetrix exon array analysis. Nucleic Acids Res. 36 (Database issue), D780-D786. doi: 10.1093/nar/ gkm779

Zhao, L., Lu, Z., Park, J. W., Zhou, Q., and Xing, Y. (2013a). GLiMMPS: robust statistical model for regulatory variation of alternative splicing using RNA-seq data. Genome Biol. 14 (7), R74. doi: 10.1186/gb-2013-14-7-r74

Zhao, P., Zou, P., Zhao, L., Yan, W., Kang, C., Jiang, T., et al. (2013b). Genetic polymorphisms of DNA double-strand break repair pathway genes and glioma susceptibility. BMC Cancer 13, 234. doi: 10.1186/14712407-13-234
Zhuang, Y., and Weiner, A. M. (1986). A compensatory base change in U1 snRNA suppresses a $5^{\prime}$ splice site mutation. Cell 46 (6), 827-835. doi: 10.1016/00928674(86)90064-4

Zuo, P., and Maniatis, T. (1996). The splicing factor U2AF35 mediates critical protein-protein interactions in constitutive and enhancer-dependent splicing. Genes Dev. 10 (11), 1356-1368. doi: 10.1101/gad.10.11.1356

Conflict of Interest: PR founded and BS is an employee of CytoGnomix. The company holds intellectual property related to information theory-based mutation analysis and validation.

EM declares that the research was conducted in the absence of any commercial or financial relationships that could be construed as a potential conflict of interest.

Copyright (C) 2020 Mucaki, Shirley and Rogan. This is an open-access article distributed under the terms of the Creative Commons Attribution License (CC BY). The use, distribution or reproduction in other forums is permitted, provided the original author(s) and the copyright owner(s) are credited and that the original publication in this journal is cited, in accordance with accepted academic practice. No use, distribution or reproduction is permitted which does not comply with these terms. 